\title{
Fractional integral representation in statistical thermodynamics of confined systems
}

\author{
Altug Sisman (1) and Jonas Fransson 10 \\ Department of Physics and Astronomy, Box 516, 75120, Uppsala University, Uppsala, Sweden
}

\begin{abstract}
(Received 6 June 2021; accepted 22 October 2021; published 11 November 2021; corrected 30 November 2021)
\end{abstract}
\begin{abstract}
Confined systems are usually treated as integer dimensional systems, like two dimensional (2D), 1D, and $0 \mathrm{D}$, by considering extreme confinement conditions in one or more directions. This approach costs piecewise representations, some limitations in confinement interval, and the deviations from the true behaviors, especially when the confinement is neither strong nor weak. In this study, fractional integral representation (FIR) is proposed as a methodology to calculate the infinite summations in statistical thermodynamics for any dimension and confinement values. FIR directly incorporates the dimension as a control variable into calculation procedures and allows us to get solutions valid for the whole confinement and dimension scales, including the fractional ones. We define the dimension of a summation and used it in the proposed FIR to calculate the partition function. The first and the higher-order FIR are introduced and high accuracy results are achieved. FIR is then extended for a generalized function to calculate thermodynamic properties directly from their fundamental expressions based on infinite sums. By using the proposed FIR approach, the thermodynamic properties of a noninteracting Maxwell-Boltzmann gas confined in an elongated rectangular domain are determined. The excess quantities induced by confinement are examined for different confinement scenarios. FIR successfully predicts the true behavior of thermodynamic properties for the whole range of confinement and dimension scales. Defining and controlling the dimension allows designing new types of thermodynamic cycles. Besides the infinite-well potential for the confinement of particles with quadratic and linear dispersion relations, quadratic and quartic confining potentials are also considered to show the success of FIR. The proposed method not only incorporates the dimension into the calculation procedures but also constitutes an application of fractional calculus in statistical thermodynamics. FIR has many potential applications especially for Bose-Einstein condensation phenomenon which inherently contains dimensional transitions.
\end{abstract}

DOI: 10.1103/PhysRevE.104.054110

\section{INTRODUCTION}

Fundamental expressions of thermodynamic properties are based on infinite summations, over either momentum or energy eigenvalues of particles, and these summations are replaced by integrals in macroscopic thermodynamics. In recent decades, however, the thermodynamics of confined systems became one of the interesting topics in nanoscale physics [1-21]. The main target of these studies is to predict the novel thermodynamic behavior of confined and low dimensional systems. In the literature, even for nanoconfined systems, integral approaches are still commonly used for the calculations of summations just by implementing the proper density of state (DOS) functions derived for integer dimensional momentum spaces [22-27]. On the other hand, a confined system undergoes a continuous dimensional transition in momentum space during the continuous change of confinement and it eventually evolves from three dimensional (3D) to $0 \mathrm{D}$ or vice versa, including the fractional ones. Nevertheless, confined systems with integer dimensions, 3D, 2D, 1D, or 0D (bulk, quantum well, wire, and dot respectively), are usually considered at

Published by the American Physical Society under the terms of the Creative Commons Attribution 4.0 International license. Further distribution of this work must maintain attribution to the author(s) and the published article's title, journal citation, and DOI. the cost of limiting the confinements [22-27]. In other words, most of the studied systems are strongly confined in one or more directions and assumed to be completely free in other directions. Otherwise, under the conditions in which the system sizes are neither much longer nor much shorter than the mean de Broglie wavelength of particles, the predictions made by integer dimensional approaches considerably deviate from the true behavior of the system. A quantum wire, for example, evolves fractional dimensional behaviors in between 1D and $0 \mathrm{D}$ as we change its length from a much longer to much shorter sizes than the mean de Broglie wavelength of particles. Besides not being able to represent the fractional dimensional behaviors, the integer dimensional approaches give distinct thermodynamic expressions for each integer dimension, instead of a single monolithic expression for all dimensions and confinement scales.

Some studies in literature, however, use either summation formulas (like Poisson, Euler-Maclaurin, and Abel-Plana) or DOS formulas based on the Weyl conjecture to calculate thermodynamic properties more precisely than that of integral approach (or DOS for integer dimensions) [22,2832]. These formulas lead to more advanced expressions for thermodynamic properties, which consider the confinement effects [1-21]. These expressions are, to some extent, able to predict the true behavior of bounded (confined) systems even if the system deviates from an integer dimensional behavior due to changes of confinement in quasifree directions. 
The expressions contain not only the bulk term, which is proportional to volume $(V)$ in the case of a 3D system, but also the lower-dimensional terms of integer order, like the ones proportional to surface area $(A)$, peripheral length $(P)$, and the number of vertices $\left(N_{\mathrm{V}}\right)$ [22,29-32]. Nevertheless, none of these advanced expressions [19,22,28-32] includes the dimension into both calculation procedures and the resulting expressions although the dimension of momentum space continuously changes during the change of confinement, e.g., $3 D \leftrightarrow 0 D$. Consequently, the formulas in the literature are not able to establish a connection between the dimension and thermodynamic properties. Also, they do not provide monolithic thermodynamic expressions which are valid for the whole confinement scale.

On the other hand, fractional calculus provides generalizations of integrals and derivatives to arbitrary order and offers many interesting and innovative approaches for different problems of physics to generalize the solutions [33-35]. Although fractional calculus has been applied to various problems of physics [34], classical thermodynamics, and heat transfer problems [36-39], there is no application yet for the calculation of the partition function and other thermodynamic properties in statistical thermodynamics. Here we show that fractional integrals have great potential as a tool to calculate infinite summations with high precision and establish a direct connection between the calculation procedure of thermodynamic properties and the dimension of momentum space. We obtain the thermodynamic expressions representing the true system behavior for the whole ranges of confinement and dimension. While the known piecewise solutions contain individual expressions for each integer dimension and are valid for certain confinement intervals, fractional integral representation (FIR) promises monolithic expressions to describe the system even under a strong dimensional transition, from 3D to $0 \mathrm{D}$, due to the strong changes in confinement. In other words, FIR can provide thermodynamic expressions valid for an arbitrary dimension, including the fractional ones, instead of the ones valid only for integer dimensions. Thus, FIR allows us to examine even the thermodynamics of systems having fractional dimensions in momentum space. When state functions become dependent on the dimension, they can be a powerful tool to analyze the changes in thermodynamic behavior during a phase transition in momentum space like the one in the Bose-Einstein condensation phenomena. There is no study yet to utilize the fractional integrals to calculate the thermodynamic properties for arbitrary dimension and confinement. This study can also provide another application possibility for fractional integrals in physics.

In this study, a noninteracting Maxwell-Boltzmann gas confined in a rectangular domain is considered to keep the primary study on FIR away from unnecessary complications. Furthermore, rectangular geometry is one of the most common geometries in nanoscale manufacturing and nanomaterials. After a short discussion about the already known methods for the partition function calculations, FIR for infinite summations is proposed and a definition of dimension for an infinite summation over a scaled variable is also introduced. It is shown that both the dimension of the partition function and the one need for FIR almost perfectly match each other. Thereby, by using the introduced dimension and the proposed FIR, the expressions for both the partition function and thermodynamic properties are obtained for arbitrary values of dimension and confinement and their variations are analyzed for the full scale of dimension.

\section{SHORT REVIEW OF THE PARTITION FUNCTION CALCULATION METHODS}

Before the derivation of FIR, the already known methods in the literature for the partition function calculations are shortly revisited and discussed in this section to reveal the advantages and the differences of FIR. One of the most fundamental and instructive problems in statistical thermodynamics is the thermodynamics of noninteracting Maxwell-Boltzmann (MB) gas confined in a rectangular domain. The energy eigenvalues $\varepsilon$ of these particles are trivial and simply obtained by solution of the Schrödinger equation for infinite potential well as

$$
\begin{gathered}
\varepsilon=\frac{h^{2}}{8 m}\left[\left(\frac{k_{1}}{L_{1}}\right)^{2}+\left(\frac{k_{2}}{L_{2}}\right)^{2}+\left(\frac{k_{3}}{L_{3}}\right)^{2}\right], \\
\left\{k_{1}, k_{2}, k_{3}\right\}=1,2,3, \ldots,
\end{gathered}
$$

where $h$ is Planck's constant, $m$ is the particle mass, $L_{1}, L_{2}, L_{3}$ are the sizes of the domain in three independent directions, and $\left\{k_{1}, k_{2}, k_{3}\right\}$ are the integer quantum state variables running from one to infinity corresponding to the three-momentum components of the particles. The single-particle partition function of a noninteracting MB gas is then given by

$$
\begin{aligned}
\zeta_{t}= & \sum_{\varepsilon} \exp \left(-\frac{\varepsilon}{k_{b} T}\right) \\
= & \sum_{k_{1}=1}^{\infty} \exp \left[-\left(\alpha_{1} k_{1}\right)^{2}\right] \\
& \times \sum_{k_{2}=1}^{\infty} \exp \left[-\left(\alpha_{2} k_{2}\right)^{2}\right] \sum_{k_{3}=1}^{\infty} \exp \left[-\left(\alpha_{3} k_{3}\right)^{2}\right],
\end{aligned}
$$

where $k_{b}$ is Boltzmann's constant, $T$ is the temperature, and $\left\{\alpha_{1}, \alpha_{2}, \alpha_{3}\right\}$ are the confinement parameters defined as $\alpha_{i}=$ $L_{c} / L_{i}=h /\left(2 \sqrt{2 m k_{b} T} L_{i}\right)$; here $L_{c}$ is the half of the most probable de Broglie wavelength of unbounded (unconfined, free) particles [5]. Note that the energy eigenvalues do not depend on the spin in the absence of a magnetic field. Therefore, the summation over the spin states is not considered here since it simply gives a constant factor, which does not play any role in either the derivations or the results. Since all thermodynamic properties can be expressed in terms of the partition function, the main task is to calculate the following summation in a precise way:

$$
\zeta(\alpha)=\sum_{k=1}^{\infty} \exp \left[-(\alpha k)^{2}\right] .
$$

For macroscopic systems, confinement parameters are extremely small $(\alpha \rightarrow 0)$ and changes of the scaled distribution function in Eq. (3) are smooth although the changes in quantum state variables are equal to unity, $\Delta k=1$. Therefore, replacing a summation operation with an integral is a very good approximation. For nanoscale systems, however, confinement parameters are not too small and some other 
TABLE I. A comparison of the contributions from different terms of PSF and the first term of the partition function.

\begin{tabular}{lccccrc}
\hline \hline$\alpha$ & $\sum_{k=1}^{\infty} \exp \left[-(\alpha k)^{2}\right]$ & $\frac{\sqrt{\pi}}{2 \alpha}$ & $-\frac{1}{2}$ & $\frac{\sqrt{\pi}}{\alpha} \sum_{s=1}^{\infty} \exp \left[-\left(\frac{\pi}{\alpha} s\right)^{2}\right]$ & $\frac{\sqrt{\pi}}{2 \alpha}-\frac{1}{2}$ & $\exp \left(-\alpha^{2}\right)$ \\
\hline 0.1 & $\mathbf{8 . 3 6 2}$ & 8.862 & -0.500 & $10^{-428}$ & $\mathbf{8 . 3 6 2}$ & 0.990 \\
1.0 & $\mathbf{0 . 3 8 6}$ & 0.886 & -0.500 & $9 \times 10^{-5}$ & $\mathbf{0 . 3 8 6}$ & 0.368 \\
2.0 & $\mathbf{0 . 0 1 8}$ & 0.443 & -0.500 & 0.075 & -0.057 & $\mathbf{0 . 0 1 8}$ \\
\hline \hline
\end{tabular}

methods need to be implemented to calculate the summations in a more precise way. One of these methods is to use one of the well-known summation formulas if the energy eigenvalues are explicitly known, as in the case considered here. For any even function, a summation from one to infinity can then be calculated by using the Poisson summation formula (PSF) as follows [5,28]:

$$
\begin{aligned}
\sum_{k=1}^{\infty} f(k)= & \int_{0}^{\infty} f(k) d k-\frac{f(0)}{2} \\
& +2 \sum_{s=1}^{\infty} \int_{0}^{\infty} f(k) \cos (2 \pi s k) d k
\end{aligned}
$$

Note that the function under the summation in Eq. (3) is scaled by confinement parameter $\alpha$ and application of Eq. (4) gives

$$
\begin{aligned}
\zeta(\alpha) & =\sum_{k=1}^{\infty} \exp \left[-(\alpha k)^{2}\right] \\
& =\frac{\sqrt{\pi}}{2 \alpha}-\frac{1}{2}+\frac{\sqrt{\pi}}{\alpha} \sum_{s=1}^{\infty} \exp \left[-\left(\frac{\pi}{\alpha} s\right)^{2}\right]
\end{aligned}
$$

It is seen that the summation formula, Eq. (4), consists of three terms: integral, zero correction, and discrete correction terms, from left to right respectively. The integral term is the term based on continuum approximation and it approaches the correct result if the confinement parameter is very small, $\alpha \ll 1$ (macrosystems). The zero-correction term constitutes some limited correction for the integral approach and these two terms together provide the correct results as long as $\alpha \leqslant \alpha_{*} \approx 1$. The discrete correction term contains another infinite sum which represents the necessary correction to the first two terms under strong confinement conditions, $\alpha>\alpha_{*} \approx 1$, because of the discrete nature of the summation process. Nevertheless, using only the first term $(s=1)$ of this discrete correction is enough to get quite correct results even in strongly confined conditions to a certain extent. Thus, depending on the nature of the function, up to a limited value of confinement, it is not usually necessary to calculate the full summation in the discrete correction (over $s$ here) which prevents obtaining the analytical results. An even better way to obtain the correct results in the case of strong confinement, $\alpha>\alpha_{*} \approx 1$, is just to consider the first term of the original summation in the left-hand side of Eq. (5). In other words, it is not necessary even to calculate the summation at all for strongly confined cases due to the strongly decaying nature of the distribution function. Therefore, the first two terms of PSF for the weak confinement regime, $\alpha \leqslant \alpha_{*} \approx 1$, and the first term (or the first two terms for even better results) of the original sum for the strong confinement regime, $\alpha>\alpha_{*} \approx 1$, are used to represent the correct results of the summation. The critical confinement value $\alpha_{*}$, which represents the transition point between weak and strong confinement representations, can easily be determined by equalizing the absolute errors of two different representations to each other [18]. Depending on the consideration of the first term or first two terms of the original sum for strongly confined conditions, the critical confinement value is obtained as $\alpha_{*}=1.24$ and $\alpha_{*}=1.01$ respectively. Because of weak and strong confinement representations, the summation formulas give only piecewise analytical expressions since otherwise, the third term of the formulas consists of also another summation that prevents obtaining analytical results. Table I clearly shows this behavior. Besides only the piecewise analytical representation ability of PSF, it does not establish any connection between the partition function and the dimension of momentum space.

Another method for the partition function calculations is to use the Weyl conjecture (Weyl's law). It provides a very precise formula for the asymptotic behavior of the number of eigenvalues of the Laplace-Beltrami operator (including the Helmholtz wave equation) for a domain of arbitrary shape [22,29-32]. Although the Weyl conjecture is an asymptotic formula, it leads to reasonably accurate results for the partition function as long as the system boundaries are smooth and the confinement is not too strong. Since the Schrödinger equation is reduced to the Helmholtz wave equation for particles confined in an infinite well potential, the Weyl conjecture allows us to calculate the partition function of noninteracting particles confined in a domain with rigid boundaries of an arbitrary shape. In other words, it is not even necessary to solve the Schrödinger equation. Instead, the partition function can be calculated by integrating over the particle energies if the Weyl conjecture is used to obtain DOS function. It is called the Weyl DOS function and, after some basic manipulations, it can be given in a compact form for dimensionless energy eigenvalues $\left(\tilde{\varepsilon}=\varepsilon / k_{b} T\right)$ with a quadratic dispersion relation $\left(\varepsilon=p^{2} / 2 m\right)$ in the case of Dirichlet boundary condition as [19]

$$
\begin{aligned}
\operatorname{DOS}_{W}(\tilde{\varepsilon})= & \frac{\pi}{4} \frac{V}{L_{c}^{3}} \sqrt{\tilde{\varepsilon}} \Theta(D-2)+\frac{(-1)^{D} \pi}{4^{D-1}} \frac{A}{L_{c}^{2}} \Theta(D-1) \\
& +\frac{(-1)^{D-1} 2}{4^{D}} \frac{P}{L_{c}} \frac{\Theta(D)}{\sqrt{\tilde{\varepsilon}}}+\frac{(-1)^{D-2} 2}{4^{D+1}} N_{V} \frac{\delta(\sqrt{\tilde{\varepsilon}})}{\sqrt{\tilde{\varepsilon}}}
\end{aligned}
$$

where $\Theta$ is the left continuous Heaviside step function, $\delta$ is the Dirac delta function, and $D$ is the integer dimension of the confinement domain. For a 3D system, the terms in Eq. (6) are called volumetric, surface, peripheral, and vertices terms, respectively, because of their proportionality 
with these geometric quantities. The high order terms, which are proportional to $\left\{A, P, \mathrm{~N}_{\mathrm{V}}\right\}$ for $3 \mathrm{D},\left\{P, \mathrm{~N}_{\mathrm{V}}\right\}$ for $2 \mathrm{D}$, and $\left\{N_{V}\right\}$ for 1D systems, are low dimensional corrections that allow calculating the partition function with very high precision for a domain of an arbitrary shape. In an infinitely large system (thermodynamic limit), the particles occupy a 3D momentum space and all the low dimensional terms become negligible in comparison with the highest dimensional one, the volumetric term. In this case, Eq. (6) consists of only the first term and leads to the well-known bulk expressions of classical thermodynamics. On the other hand, if the system is finite and $L_{c}$ is not negligible in comparison with the system sizes, then the low dimensional terms make considerable contributions allowing us to predict the true system behavior. In this case, the system is called a bounded 3D system. Due to the wave nature of particles, boundary modes are forbidden in a finite system and the low dimensional terms basically exclude the contributions of boundary modes which are incorrectly considered by the 3D term, the first term. In other words, the low dimensional terms are the corrections representing the deviations from ideal 3D behavior due to confinement. Nevertheless, the Weyl conjecture (or Weyl DOS) does not establish a connection between these terms and the dimension of momentum space, except the step functions which lead to piecewise and integer dimensional representations. If the system is strongly confined in one direction, then the corrections of low dimensional terms are not sufficient to predict the true system behavior anymore and it becomes necessary to switch from 3D representation to the 2D one in Eq. (6). This transition point corresponds to the condition of $V \approx A L_{c}$. Beyond this point, $V<A L_{c}$, the particles are free to occupy 2D momentum space while they stay at the ground state of the momentum component in a strongly confined direction. Therefore, the system is considered as a bounded 2D system with corrections from lower dimensional contributions. Similarly, this approach works well until the confinement in the second direction becomes also strong, $A \approx P L_{c}$. Beyond that condition, $A<P L_{c}$, the 1D form of Eq. (6) has to be used to predict the true system behavior. Finally, when the confinement is strong in all directions, $P \approx N_{V} L_{c}$, then only $0 \mathrm{D}$ representation is applied. Note that the partition function calculated from Eq. (6) can also be obtained if the first two terms of PSF are used for each direction when the momentum eigenvalues are explicitly known, like in the case of a rectangular domain.

Consequently, the Weyl conjecture gives different thermodynamic expressions for each integer dimension. It does not establish a connection between the dimension of momentum space, including the fractional ones, and a DOS function as well as the resulting thermodynamic expressions. In other words, neither the Weyl conjecture, Eq. (6), nor PSF, Eq. (4), offer thermodynamic expressions as a continuous function of dimension and they do not predict the true thermodynamic behavior of a system for the whole confinement range. None of them are able to represent thermodynamic behavior under continuous dimensional transitions $(3 D \leftrightarrow 2 D \leftrightarrow 1 D)$ that occurred during the continuous and strong changes in confinement.

For more general representations, which are valid for arbitrary confinement and dimensions including the fractional ones, a different approach is developed and proposed here by implementing the fractional integrals for the calculation of infinite summations of scaled functions. The dimensions of fractional integrals are also defined by considering the infinite summations. The following section gives the derivation of this approach to calculate the infinite sums of scaled functions in statistical physics.

Before we begin the derivations and calculations of FIR for MB statistics, a short discussion about the applicability limits of MB statistics is justified. The necessary and sufficient condition to make use of Maxwell-Boltzmann (MB) statistics is just to keep the occupation number of the ground state, $N_{g}$, much smaller than unity, $N_{g} \ll 1$, to neglect the nature of quantum statistics while keeping the total number of particles in the opposite condition, $N \gg 1$. Thus, $N_{g}=$ $N \exp \left(-\tilde{\varepsilon}_{0}\right) / \zeta_{t} \ll 1$ which leads to $1 \ll N \ll \zeta_{t} \exp \left(\tilde{\varepsilon}_{0}\right)$. Here $\tilde{\varepsilon}_{0}=\varepsilon_{0} / k_{b} T$ is the dimensionless ground state energy which is $\tilde{\varepsilon}_{0}=\alpha_{1}^{2}+\alpha_{2}^{2}+\alpha_{3}^{2}$ for quadratic dispersion relation and a rectangular confinement domain considered here. If the system is strongly confined in all directions $(0 D)$, then $\zeta_{t} \rightarrow$ $\exp \left(-\tilde{\varepsilon}_{0}\right)$ and $N=N_{g}$. Therefore, it is not possible to ensure both $N_{g} \ll 1$ and $N \gg 1$ for a OD MB system. To make use of MB statistics, we have to consider very weakly confined or even unconfined systems in at least one direction. Thus, the minimum dimension of a finite size MB system is one dimension. As long as one of the confinements is small enough, $\alpha_{1} \ll 1$, (here $\alpha_{1}$ is chosen), it is possible to keep both $N_{g} \ll 1$ and $N \gg 1$ independent of whether the confinements in the other two directions are weak $\left\{\alpha_{2}, \alpha_{3}\right\} \ll 1$ or strong, $\left\{\alpha_{2}, \alpha_{3}\right\}>1$. Therefore, a rectangular domain elongated in one direction (direction-1 here) is chosen and only the changes of confinements in transverse directions $\left(\alpha_{2}\right.$ and $\left.\alpha_{3}\right)$ are considered while the longitudinal one is kept very small, $\alpha_{1} \ll 1$. Note that, however, if the system is ergodic, the time averages are equal to ensemble averages, then it is also possible to consider even one-particle systems in MB statistics.

\section{FRACTIONAL INTEGRAL REPRESENTATION OF THE PARTITION FUNCTION}

\section{A. Derivation of the first order (ground state) FIR}

The partition function in Eq. (3) can identically be expressed in terms of an integral by employing the Dirac delta function $\delta$ as

$$
\begin{aligned}
\zeta(\alpha) & =\sum_{k=1}^{\infty} \exp \left[-(\alpha k)^{2}\right] \\
& =\sum_{n=1}^{\infty} \int_{0}^{\infty} \delta(k-n) \exp \left[-(\alpha k)^{2}\right] d k .
\end{aligned}
$$

We know that when $\alpha \rightarrow 0$ (deconfinement or infinitely large system limit) the partition function converges into an integral since the spectral space of the function, $\exp \left[-(\alpha k)^{2}\right]$, becomes a 1D continuum space due to infinitely dense sampling. On the other hand, when $\alpha \rightarrow \infty$ (complete confinement or infinitesimally small system limit) the partition function consists of only the first term and the spectral space 
of the function becomes a $0 \mathrm{D}$ point space as a result of infinitely sparse sampling. In mathematical expression,

$$
\zeta(\alpha)=\sum_{n=1}^{\infty} \int_{0}^{\infty} \delta(k-n) \exp \left[-(\alpha k)^{2}\right] d k=\left\{\begin{array}{l}
\alpha \underset{(D \rightarrow 1)}{\rightarrow \rightarrow} 0 \Rightarrow \int_{0}^{\infty} \exp \left[-(\alpha k)^{2}\right] d k=\frac{\sqrt{\pi}}{2 \alpha} \\
\alpha \underset{(D \rightarrow 0)}{\rightarrow \rightarrow} \infty \Rightarrow \int_{0}^{\infty} \delta(k-1) \exp \left[-(\alpha k)^{2}\right] d k=\exp \left(-\alpha^{2}\right)
\end{array}\right.
$$

By considering the limit representation of the Dirac delta function [40], Eq. (7) can be rewritten in the following form as well:

$$
\zeta(\alpha)=\lim _{D \rightarrow 0} \sum_{n=1}^{\infty} \int_{0}^{\infty} D|2(k-n)|^{D-1} \exp \left[-(\alpha k)^{2}\right] d k .
$$

Asymptotic results shown in Eq. (8) can directly be obtained if the limit operation on $D$ and summation over $n$ in Eq. (9) are changed from $\{D \rightarrow 0$ and $n=1,2,3, \ldots \infty\}$ to $\{D=0, \ldots, 1$ and $n=1\}$. In other words, instead of fixing the value of $D$ as zero and letting $n$ free to take the values in the interval of $\{1,2,3 \ldots, \infty\}$, we fix the value of $n$ as $n=1$ and let $D$ take the values in the interval of $\{0, \ldots, 1\}$. In that case, the partition function can precisely be written, at least for the asymptotic cases $(D \rightarrow 0 \& D \rightarrow 1)$, as

$$
\begin{aligned}
& \left\{\begin{array}{c}
\text { first order } \\
\text { representation }
\end{array}\right\}: \zeta(\alpha) \approx{ }_{1} \zeta(\alpha) \\
& =\int_{0}^{\infty} D|2(k-1)|^{D-1} \exp \left[-(\alpha k)^{2}\right] d k \\
& =\int_{0}^{\infty} \delta_{D}(k-1) \exp \left[-(\alpha k)^{2}\right] d k
\end{aligned}
$$

where $\delta_{D}(x)=D|2 x|^{D-1}$ is used here as a free form of the Dirac delta function which goes to the known (true) Dirac delta function when $D \rightarrow 0$ while it becomes unity in the opposite limit, $D \rightarrow 1$. When the confinement goes to infinity (absolute confinement), $\alpha \rightarrow \infty(D \rightarrow 0)$, the partition function consists of only the contribution of the ground state, state $1(k=1)$, and the contributions from higher states become completely negligible. In other words, the partition function shrinks towards the ground state and this behavior is performed by $\delta_{D}(k-1)$ in Eq. (10b) where state 1 stands for the concentration point of the integration process in case of absolute confinement $(D \rightarrow 0)$. Therefore, Eq. (10) is called here first order representation or ground state representation. The subscript " 1 " on $\zeta$ indicates the order of representation. This reduction of the integration process into the contribution from state 1 is called here "condensation of integration" by using the analogy between the processes of Bose-Einstein condensation and integration. In the opposite limit, $\alpha \rightarrow 0(D \rightarrow 1)$, the classical first order integral approach is recovered as a result of the properties of $\delta_{D}$. At this point, the following question may arise: Is Eq. (10) true only for the asymptotic cases, $\alpha \rightarrow 0$ and $\alpha \rightarrow \infty$, or also for any values of $\alpha$ as long as $D(\alpha)$ can be determined in some way? The answer is yes, and the detailed recipe for the determination of $D(\alpha)$ is proposed in the following Sec. III B. Therefore, Eq. (10) constitutes the base of FIR for the partition function and provides a representation in the full range of confinement by a single expression. It gives the exact results for the asymptotic cases and high accuracy results for the intermediate values of the confinement. The maximum error occurs only around $\alpha=0.3$ in the order of less than $2.5 \%$ for first order representation. Detailed analysis about the success of Eq. (10) to represent the partition function is given in Sec. III C.

Note that $\delta_{D}(x)$ is different from what it is called the fractional Dirac delta function $\delta_{D}^{F}(x)$ with dimension $D$, which is obtained by using the following the left-handed Liouville fractional integral [41]:

$\delta_{D}^{F}(x)=\frac{D}{\Gamma(1+D)} \int_{-\infty}^{x}(x-s)^{D-1} \delta(s) d s=\frac{D x^{D-1}}{\Gamma(1+D)} \Theta(x)$,

where $\Gamma$ represents the Gamma function. The differences between the behaviors of $\delta_{D}(x)$ and $\delta_{D}^{F}(x)$ are given as follows:

$$
\begin{array}{ll}
\lim _{D \rightarrow 0} \int_{0}^{\infty} \delta_{D}(x) d x=\frac{1}{2}, & \lim _{D \rightarrow 1} \int_{0}^{x} \delta_{D}\left(x^{\prime}\right) d x^{\prime}=x, \\
\lim _{D \rightarrow 0} \int_{0}^{\infty} \delta_{D}^{F}(x) d x=1, & \lim _{D \rightarrow 1} \int_{0}^{x} \delta_{D}^{F}\left(x^{\prime}\right) d x^{\prime}=x \Theta(x) .
\end{array}
$$

Note that integral of $\delta_{D}(x)$ gives the correct result in the case of $D \rightarrow 0$ while $\delta_{D}^{F}(x)$ leads to a result twice as large. In the opposite case $(D \rightarrow 1)$, the integral of $\delta_{D}(x)$ is correct only for positive $x$ values while $\delta_{D}^{F}(x)$ leads to the correct results for any value of $x$. This is because of the fractional integral form used in Eq. (11) where the integrand contains the upper limit of the integral. Since the integration in Eq. (10) takes only in the positive interval $(0, \infty), \delta_{D}(x)$ represents the correct form to calculate the partition function due to its behaviors given in Eq. (12a). The integral in Eq. (10) is closely related to the Riemann-Liouville fractional integrals. It can be split into two parts:

$$
\begin{aligned}
\int_{0}^{\infty} & \delta_{D}(k-1) \exp \left[-(\alpha k)^{2}\right] d k \\
= & 2^{D-1} D\left[\int_{0}^{1}(1-k)^{D-1} \exp \left[-(\alpha k)^{2}\right] d k\right. \\
& \left.+\int_{1}^{\infty}(k-1)^{D-1} \exp \left[-(\alpha k)^{2}\right] d k\right] .
\end{aligned}
$$

The right-hand side of Eq. (13) can then be rewritten in terms of Riemann-Liouville fractional integrals as follows:

$$
\begin{aligned}
& \int_{0}^{\infty} \delta_{D}(k-1) \exp \left[-(\alpha k)^{2}\right] d k \\
& \quad=2^{D-1} \Gamma(1+D)\left({ }_{0}^{1} \mathrm{I}_{D}+{ }_{1}^{\infty} \mathrm{I}_{D}\right) \exp \left[-(\alpha k)^{2}\right],
\end{aligned}
$$

where ${ }_{0}^{1} I_{D}$ and ${ }_{1}^{\infty} I_{D}$ are the left-handed Riemann and the righthanded Liouville fractional integral operators respectively [34]. In other words, $\delta_{D}$ allows expressing the right-hand side of Eq. (14) by a single integral operator on the left-hand side. Therefore, Eq. (10b) is the proposed FIR for the partition function. For simplicity, FIR of the partition function is 
symbolized here by $\operatorname{FIR}_{1}(\zeta)$ if it is based on the first-order approach. It can even analytically be solved as follows:

$$
\begin{aligned}
\operatorname{FIR}_{1}(\zeta)= & \int_{0}^{\infty} \delta_{D}(k-1) \exp \left[-(\alpha k)^{2}\right] d k \\
= & 2^{D-1}{ }_{2} \mathrm{~F}_{2}\left[\left(\frac{1}{2}, 1\right),\left(\frac{1+D}{2}, \frac{2+D}{2}\right),-\alpha^{2}\right] \\
& +\frac{\exp \left(-\alpha^{2}\right)}{2 \alpha^{D}} \Gamma(1+D) \mathrm{FU}\left(\frac{D}{2}, \frac{1}{2}, \alpha^{2}\right),
\end{aligned}
$$

where ${ }_{2} F_{2}$ and FU are the generalized hypergeometric function and the second kind of confluent hypergeometric function respectively. For the asymptotic cases of $D=1$ and $D=$ 0 , Eq. (15b) reduces to its simplest forms as $\sqrt{\pi} / 2 \alpha$ and $\exp \left(-\alpha^{2}\right)$ respectively due to the behaviors of ${ }_{2} F_{2}$ and FU for asymptotic values of $D$. Note that when $D \rightarrow$ $1,{ }_{2} F_{2} \rightarrow \sqrt{\pi} \operatorname{Erf}(\alpha) / 2 \alpha$ and $\mathrm{FU} \rightarrow \exp \left(\alpha^{2}\right) \sqrt{\pi}[1-\operatorname{Erf}(\alpha)]$ while ${ }_{2} F_{2} \rightarrow \exp \left(-\alpha^{2}\right)$ and $\mathrm{FU} \rightarrow 1$ in the case of $D \rightarrow 0$.

\section{B. Definition of dimension for the first order FIR}

In order to calculate the partition function by FIR, given in Eqs. (10b) and (15b), the relation between the dimension and confinement, $D(\alpha)$, has to be known. A conjecture for the dimension of the partition function can be made as follows. The length (magnitude) of the partition function, $L(\alpha)$, is defined as follows by choosing the first term of the partition function as a measure, $m(\alpha)$ :

$$
L(\alpha)=\frac{\zeta(\alpha)}{m(\alpha)}=\frac{\sum_{k=1}^{\infty} \exp \left[-(\alpha k)^{2}\right]}{\exp \left(-\alpha^{2}\right)} .
$$

Similarly, the length of the classical partition function, represented by the first-order integral approach, can also be defined by using the same measure of $m(\alpha)$ as

$$
L_{c l}(\alpha)=\frac{\zeta_{c l}(\alpha)}{m(\alpha)}=\frac{\int_{0}^{\infty} \exp \left[-(\alpha k)^{2}\right] d k}{\exp \left(-\alpha^{2}\right)} .
$$

The dimension $D(\alpha)$ can then simply be conjectured as a relation between $L(\alpha)$ and $L_{c l}(\alpha)$ in the following form:

$$
L(\alpha)=L_{c l}(\alpha)^{D(\alpha)} .
$$

It is easily seen that $\zeta(\alpha) \rightarrow \zeta_{c l}(\alpha)$ when $D \rightarrow 1$, while $\zeta(\alpha) \rightarrow \exp \left(-\alpha^{2}\right)$ in the case of $D \rightarrow 0$. Equations (16) and (17) are essentially the inverse of the occupation probabilities of the ground state in the true (discrete) and classical representations respectively. In other words, the dimension can be expressed in a more refined form in terms of probabilities as

$$
\begin{aligned}
& p_{1}(\alpha)=p_{1, c l}(\alpha)^{\zeta D(\alpha)} \Rightarrow{ }_{1}^{\zeta} D(\alpha)=\frac{\ln \left[p_{1}(\alpha)\right]}{\ln \left[p_{1, c l}(\alpha)\right]} \\
& =\frac{\ln \left[\frac{\exp \left(-\alpha^{2}\right)}{\zeta(\alpha)}\right]}{\ln \left[\frac{\exp \left(-\alpha^{2}\right)}{\zeta_{c l}(\alpha)}\right]}
\end{aligned}
$$

Equation (19) gives the definition of the dimension for the partition function. The subscript " 1 " on $D$ indicates that the dimension is gauged to the state 1 . Therefore, $D=0$ if the partition function consists of only the contribution from state 1 , (ground state). When $D=1$, then all states contribute to the partition function with equal probability. In this sense,

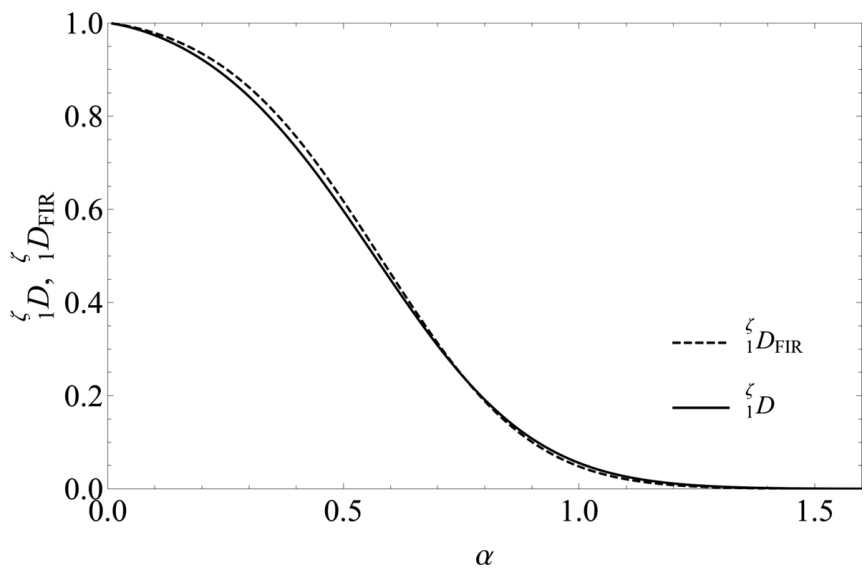

FIG. 1. A comparison between the dimension of the partition function ${ }_{1}^{\zeta} D$, Eq. (19), and the dimension of FIR for the partition function, ${ }_{1}^{\zeta} D_{\mathrm{FIR}}$, which is obtained from the equality of Eqs. (15b) and (3).

dimension is related to the form of the occupation probability distribution; 1D and 0D correspond to flat and infinitely sharp distributions respectively. Note that $\ln p_{1}$ is the Shannon information of ground state [42]. In other words, dimension in Eq. (19) is just the ratio of ground state contributions to Shannon information in discrete and classical representations. Because of the power-law relation considered in between $p_{1}$ and $p_{1, c l}$, the dimension given by Eq. (19) is also equivalent to Higuchi dimension [43] given by

$$
{ }_{1}^{\zeta} D(\alpha)=\frac{d \ln \left[p_{1}\left(p_{1, c l}\right)\right]}{d \ln \left(p_{1, c l}\right)} .
$$

To check the accuracy of ${ }_{1}^{\zeta} D$ given by Eq. (19), it is compared with ${ }_{1}^{\zeta} D_{\mathrm{FIR}}$, the one needed for Eq. (15b). ${ }_{1}^{\zeta} D_{\mathrm{FIR}}$ is obtained by making Eq. (3) equal to Eq. (15b) and using a numerical iterative inverse solution while the true partition function, Eq. (3), is calculated by numerical summation. In this way, the predictions of Eq. (19) are compared with ${ }_{1}^{\zeta} D_{\text {FIR }}$. Figure 1 shows that although these dimensions are obtained in totally different ways they are remarkably in very good agreement. The maximum difference between these dimension functions is just 0.02 . These functions cross each other around $\alpha \approx 0.76$. Except at the crossing point, ${ }_{1}^{\zeta} D_{\mathrm{FIR}}$ either slightly over- or underestimates the dimension. This is because FIR is an approach for integral representation, but not the exact solution. In other words, Eqs. (15a) and (15b) are simply approximations, however, providing the high precision results given in Sec. III C. Note that for a sum starting from 0 instead of 1 , the first term, used as a measure $m(\alpha)$, may not depend on the scale parameter $\alpha$ and this causes ill predictions for dimension. In such a case, we can simply separate the first term and consider the rest of the sum to define the dimension for and apply FIR without any problem. This way can also be preferred just to obtain better FIR results, as it is chosen in Appendix B.

To get a fully analytical representation, we also need to know the dimension analytically to use it in the analytical results of FIR, like Eq. (15b), as a control variable. 

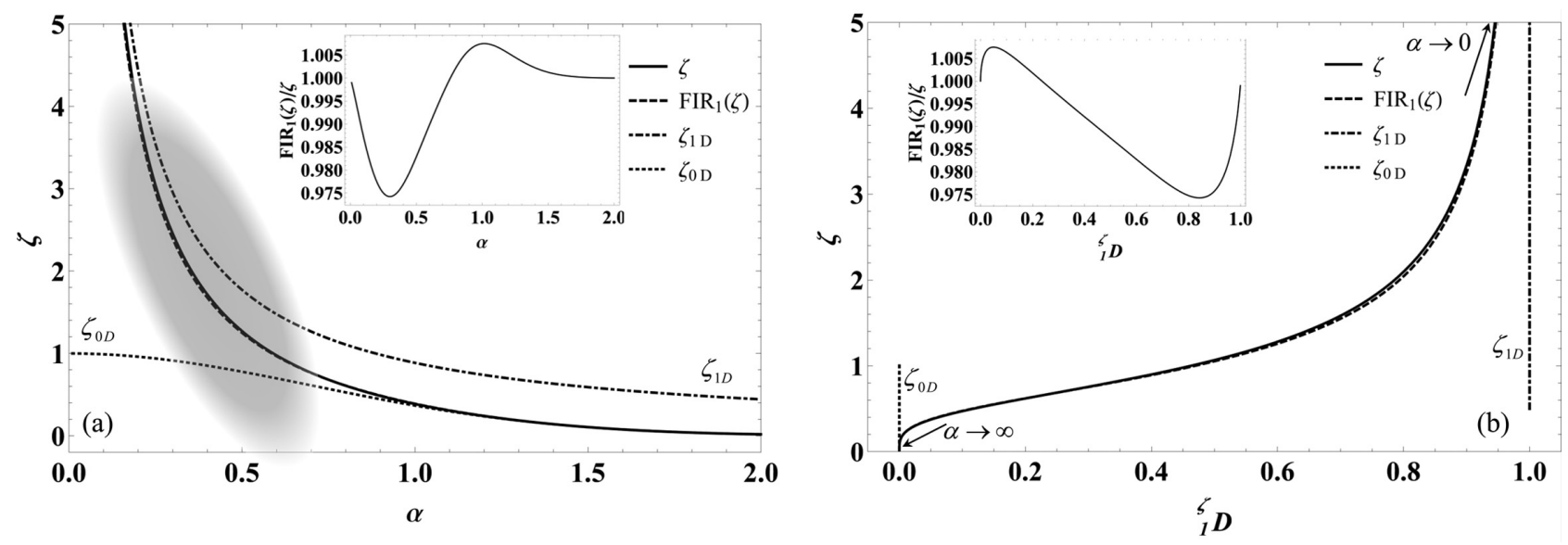

FIG. 2. Variations of the partition function (solid curve) $\zeta$, $\operatorname{FIR}_{1}(\zeta)$ (dashed curve), 1D (dot-dashed) and 0D (dotted) representations of partition functions, $\zeta_{1 D}=\sqrt{\pi} / 2 \alpha$ and $\zeta_{0 D}=\exp \left(-\alpha^{2}\right)$, with confinement $\alpha$ (a) and dependencies of the same quantities on dimension ${ }_{1}^{\zeta} D$ (b). Inset figures show the variations of accuracy, $\operatorname{FIR}_{1}(\zeta) / \zeta$, with confinement and dimension respectively.

Fortunately, it is possible to express the dimension in an analytical form quite successfully even if the term $\zeta(\alpha)$ in Eq. (19) is approximated by the first two terms of PSF, Eq. (4), for small values of $\alpha$ and by the first two terms of the partition function itself for large values of $\alpha$. In other words, despite $\zeta(\alpha)$ being represented by a continuous function given by $\operatorname{FIR}_{1}(\zeta)$, Eq. (15b), we can use the following piecewise function for $\zeta(\alpha)$ in Eq. (19) to calculate the dimension, ${ }_{1}^{\zeta} D(\alpha)$ :

$$
\zeta(\alpha)=\sum_{k=1}^{\infty} \exp \left[-(\alpha k)^{2}\right] \approx \begin{cases}\zeta_{L}(\alpha)=\int_{0}^{\infty} \exp \left[-(\alpha k)^{2}\right] d k-\frac{1}{2}=\frac{\sqrt{\pi}}{2 \alpha}-\frac{1}{2}, & \alpha \leqslant \alpha_{*} \\ \zeta_{H}(\alpha)=\exp \left(-\alpha^{2}\right)+\exp \left(-4 \alpha^{2}\right), & \alpha \geqslant \alpha_{*}\end{cases}
$$

The value of $\alpha_{*}$ can easily be found by the numerical solution of $\zeta_{H}(\alpha)=\zeta_{L}(\alpha)$ as $\alpha_{*}=1.008$, or more generally by $\left|\zeta(\alpha)-\zeta_{L}(\alpha)\right|=\left|\zeta_{H}(\alpha)-\zeta(\alpha)\right|$ if there is no intersect. Therefore, Eq. (19) can be simplified and even analytically be expressed as

$$
{ }_{1 a}^{\zeta} D(\alpha) \approx\left\{\begin{array}{ll}
1-\frac{\ln \left(1-\frac{\alpha}{\sqrt{\pi}}\right)}{\ln \left(\frac{2 \alpha}{\sqrt{\pi}}\right)-\alpha^{2}}, & \alpha \leqslant \alpha_{*}=1.008 \\
\frac{\ln \left[1+\exp \left(-3 \alpha^{2}\right)\right]}{\alpha^{2}-\ln \left(\frac{2 \alpha}{\sqrt{\pi}}\right)}, & \alpha>\alpha_{*}=1.008
\end{array} .\right.
$$

The maximum relative error of Eq. (22) occurs at $\alpha=\alpha_{*}$ as $0.6 \%$. Thus, it provides a very good analytical representation of dimension ${ }_{1}^{\zeta} D(\alpha)$ for $\operatorname{FIR}_{1}(\zeta)$.

Instead of using Eq. (21) for direct representation of $\zeta(\alpha)$, we use it only for the calculation of dimension, ${ }_{1}^{\zeta} D(\alpha)$, and then use the dimension in $\operatorname{FIR}_{1}(\zeta)$, Eq. (15b), to represent $\zeta(\alpha)$. It may seem to be an indirect way for the calculation of $\zeta(\alpha)$, however, in this way, dimension is inherently incorporated into the calculation procedure of the partition function and so are the other thermodynamic properties. This makes the dimension a control parameter on the calculation process which allows using the integral representation for the whole confinement and dimension scales including fractional ones. Another advantage of FIR is providing information about the dimension and to use it as a control variable also on thermodynamic state functions.

\section{Accuracy of first order FIR for the partition function}

If the dimension given in Eq. (22) is used in $\operatorname{FIR}_{1}(\zeta)$, Eq. (15b), we get a continuous representation of the partition function for the whole confinement scale, $0<\alpha<\infty$. This allows us to represent thermodynamic properties by a single and unified expression instead of using different expressions valid only for discrete integer dimensions of 1 and 0 (unconfined and completely confined). The plots in Fig. 2(a) demonstrate that while the $0 \mathrm{D}$ and $1 \mathrm{D}$ results deviate considerably from the exact solutions for weak and strong confinement conditions respectively, the $\mathrm{FIR}_{1}(\zeta)$ result agrees very well with the exact ones for the whole interval of confinement. Particularly, the gray zone on Fig. 2(a) indicates the region where neither 1D nor 0D approaches are able to represent the true behavior. The maximum error of $\operatorname{FIR}_{1}(\zeta)$ appears around $\alpha=0.3$ as $2.5 \%$. Furthermore, $\operatorname{FIR}_{1}(\zeta)$ is also calculated by using the exact expression of dimension, Eq. (19), and compared with the one using Eq. (22). It is seen that the analytical approach for dimension causes only $0.03 \%$ maximum relative difference in $\operatorname{FIR}_{1}(\zeta)$ around $\alpha=$ 0.3 , which is totally negligible and shows the success of Eq. (22). Variations of the same quantities with dimension are given in Fig. 2(b). In dimension space, it is explicitly seen that $1 \mathrm{D}$ and $0 \mathrm{D}$ approaches are able to represent the true behaviors only for the asymptotic cases of confinement, $\alpha \rightarrow 0$ and $\alpha \rightarrow \infty$, respectively.

Since we are now able to express the partition function as a function of dimension for the whole range of confinement 


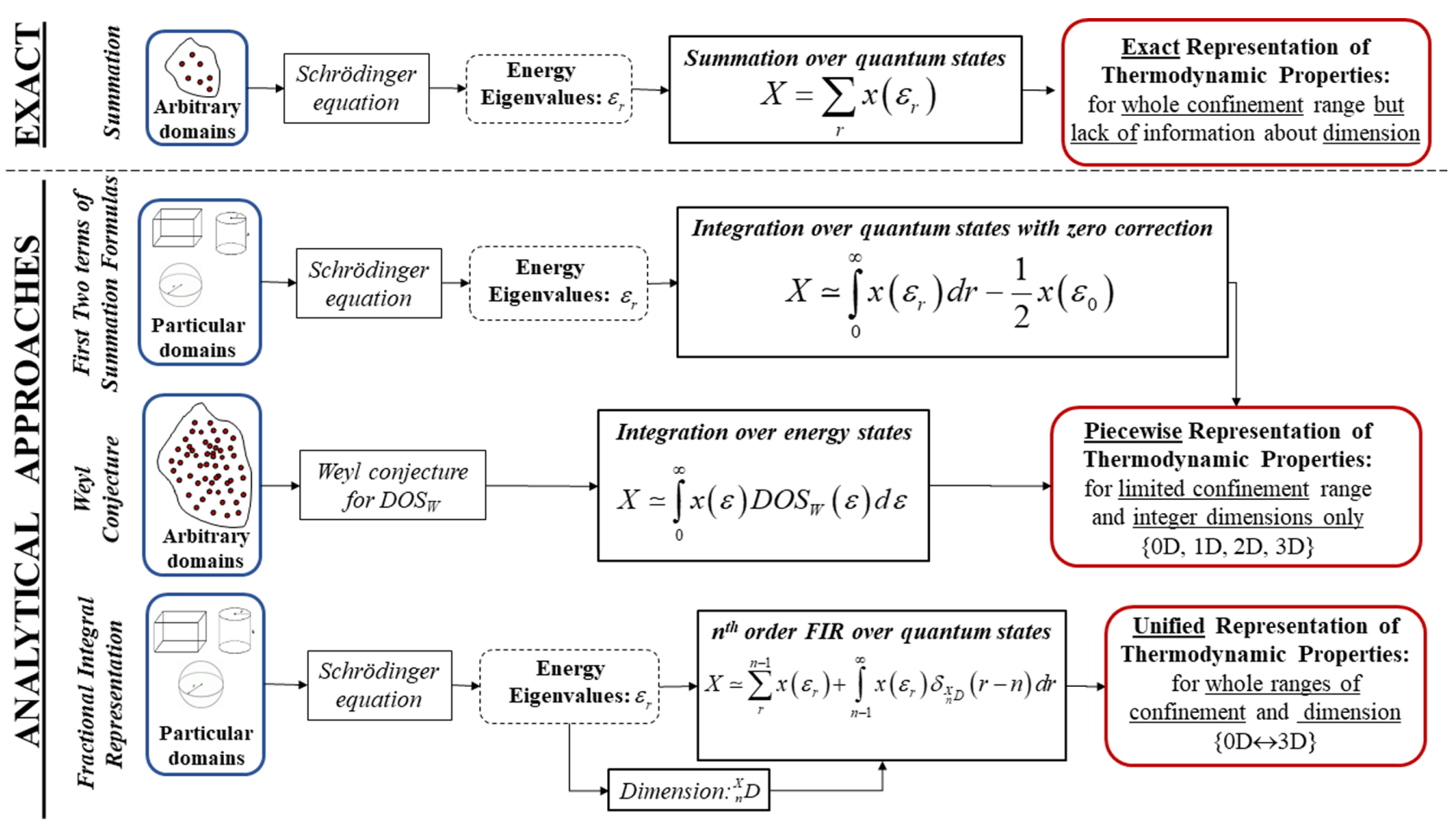

FIG. 3. A schematic comparison of FIR with already known methods for the calculation of thermodynamic properties.

by FIR, it is possible to obtain the total partition function just by multiplication of its FIR expression in each direction; see Eq. (2). After the total partition function is determined, it is possible to obtain the unified expressions of thermodynamic state functions, which are valid for the whole confinement range. It should be noted that similar to other summation formulas, FIR works when the energy eigenvalues are explicitly known, e.g., like the ones for a rectangular domain.

Despite its success for the partition function, the first order FIR may not be sufficient for the summations consisting of high order moments of energy multiplied by the distribution function, like $\sum(\alpha k)^{4} \exp \left[-(\alpha k)^{2}\right]$. Depending on the order of the moment and the value of confinement, the dimension may take even the unphysical negative values and it happens when simply the order of FIR is insufficient to represent the considered sum. This situation can easily be overcome just by selecting higher $n$ values, going to higher-order representations. Therefore, there is a need to consider the second or higher-order (excited state) FIR to directly calculate this kind of summations which appear in statistical thermodynamics as fundamental expressions of some quantities like internal energy, heat capacity, etc. Derivation of high order FIR for common summations in statistical thermodynamics is given in Appendix A. It is found that the second order is the minimum order to calculate the quantities consisting of the second order moment of energy, like heat capacity. Thus, the second order FIR is chosen here to calculate all thermodynamic properties directly from their fundamental expressions.

On the other hand, it is clear that thermodynamic quantities can indirectly be determined by using the partition function, instead of using their expressions based on infinite summations. Therefore, once $\operatorname{FIR}_{1}(\zeta)$ is determined, the other thermodynamic properties can be derived from $\operatorname{FIR}_{1}(\zeta)$. In that case, however, derivations and expressions of thermodynamic properties become unnecessarily complicated due to the dimensional dependence of $\operatorname{FIR}_{1}(\zeta)$. Note that dimension also depends on confinement and so the temperature and sizes of the system. Because of this reason, it is always much easier to calculate the properties by using their fundamental expressions. This is why the high order FIR in Appendix A is needed. Similarly, we derive and examine the higher-order FIR particularly for the partition function in the following Sec. III D, not only for a better representation (higher accuracy) of $\zeta$ but also for a complete and generalized recipe. Furthermore, to show its consistent agreement with the exact results, FIR of partition functions for quadratic and quartic confining potentials are also considered and examined in Appendix B.

A schematic comparison of both FIR and the known methods for the calculation of thermodynamic properties is given in Fig. 3. The main advantage of FIR is that it incorporates the dimension into calculation steps and therefore allows us to obtain unified expressions valid for the whole confinement and dimension scales, including the fractional ones, besides raising awareness about the dimension. In its present form, the only disadvantage of FIR is its limited applicability only for some particular domains for which energy eigenvalues are explicitly known. On the other hand, one of the most common manufactured structures in nanoscale is already rectangular ones, where FIR can easily be applied.

\section{Extension of FIR to high order (excited state) representations: $\operatorname{FIR}_{n}(\zeta)$ and ${ }_{n}^{\zeta} D$}

To obtain more precise forms of $\operatorname{FIR}_{n}(\zeta)$, an excited state (state $n$ ) has to be chosen as a condensation point of 
integration. Therefore, for extension of $\operatorname{FIR}_{n}(\zeta)$ to any order of $n, \zeta(\alpha)$ has to split into two parts,

$$
\begin{aligned}
\zeta(\alpha)= & \sum_{k=1}^{\infty} \exp \left[-(\alpha k)^{2}\right]=\sum_{k=1}^{n-1} \exp \left[-(\alpha k)^{2}\right] \\
& +\sum_{k=n}^{\infty} \exp \left[-(\alpha k)^{2}\right], \quad n \geqslant 1 .
\end{aligned}
$$

Then FIR is applied just for the second summation and the following expression is obtained for $\operatorname{FIR}(\zeta)$ by extending the idea in Eq. (10b):

$$
\begin{aligned}
& \left\{\begin{array}{c}
n \text {th order } \\
\text { representation }
\end{array}\right\}: \quad \operatorname{FIR}_{n}(\zeta) \\
& =\sum_{k=1}^{n-1} \exp \left[-(\alpha k)^{2}\right] \\
& \quad+\int_{n-1}^{\infty} \exp \left[-(\alpha k)^{2}\right] \delta_{D}(k-n) d k, \quad n \geqslant 1,
\end{aligned}
$$

where $n$ is a positive integer number referring to the order of representation and it also refers to the chosen state on which condensation of the integration process is tuned. Note that the summation term in Eq. (24) vanishes for the first-order case, $n=1$. Similar to Eq. (14), the integral term in Eq. (24) has the following relation with fractional integral operators:

$$
\begin{aligned}
& \int_{n-1}^{\infty} \exp \left[-(\alpha k)^{2}\right] \delta_{D}(k-n) d k \\
& \quad=2^{D-1} \Gamma(1+D)\left({ }_{n-1}^{n} \mathrm{I}_{D}+{ }_{n}^{\infty} \mathrm{I}_{D}\right) \exp \left[-(\alpha k)^{2}\right],
\end{aligned}
$$

where ${ }_{n-1}^{n} \mathrm{I}_{D}$ and ${ }_{n-1}^{\infty} \mathrm{I}_{D}$ are the right-handed Riemann and the left-handed Liouville fractional integral operators respectively [34]. Therefore, Eq. (24) is the general form of FIR for the partition function and it has a semianalytical solution given by

$$
\begin{aligned}
\operatorname{FIR}_{n}(\zeta)= & \sum_{k=1}^{n-1} \exp \left[-(\alpha k)^{2}\right]+\int_{0}^{\infty} \exp \left[-(\alpha k)^{2}\right] \delta_{D}(k-n) d k \\
& -\int_{0}^{n-1} \exp \left[-(\alpha k)^{2}\right] \delta_{D}(k-n) d k
\end{aligned}
$$

$$
\begin{aligned}
= & \sum_{k=1}^{n-1} \exp \left[-(\alpha k)^{2}\right]+{ }_{0}^{\infty} \Im_{n}^{D} \exp \left[-(\alpha k)^{2}\right] \\
& -{ }_{0}^{n-1} \Im_{n}^{D} \exp \left[-(\alpha k)^{2}\right] \\
= & \sum_{k=1}^{n-1} \exp \left[-(\alpha k)^{2}\right]+2^{D-1} n^{D}{ }_{2} \mathrm{~F}_{2}\left[\left(\frac{1}{2}, 1\right),\right. \\
& \left.\times\left(\frac{1+D}{2}, \frac{2+D}{2}\right),-(\alpha n)^{2}\right] \\
& +\frac{\exp \left[-(\alpha n)^{2}\right]}{2 \alpha^{D}} \Gamma(1+D) \mathrm{FU}\left[\frac{D}{2}, \frac{1}{2},(\alpha n)^{2}\right] \\
& -{ }_{0}^{n-1} \Im_{n}^{D} \exp \left[-(\alpha k)^{2}\right] ;
\end{aligned}
$$

here FIR operators ${ }_{0}^{\infty} \Im_{n}^{D}$ and ${ }_{0}^{n-1} \Im_{n}^{D}$ are introduced for brevity. The last term in Eq. (26c), the one containing the operator ${ }_{0}^{n-1} \mathfrak{\Im}_{n}^{D}$, should be calculated numerically and it becomes zero only for "the first order representation," $n=1$, for which Eq. (26c) reduces to Eq. (15b).

The dimension for this $n$th order representation is defined by considering the summation from $n$ to infinity in Eq. (23) as

$$
{ }_{n}^{\zeta} D(\alpha)=\frac{\ln \left[p_{n}(\alpha)\right]}{\ln \left[p_{n, c l}(\alpha)\right]}=\frac{\ln \left[\frac{\exp \left[-(\alpha n)^{2}\right]}{\sum_{k=n}^{\infty} \exp \left[-(\alpha k)^{2}\right]}\right]}{\ln \left[\frac{\exp \left[-(\alpha n)^{2}\right]}{\int_{n-1}^{\infty} \exp \left[-(\alpha k)^{2}\right] d k}\right]} .
$$

The classical representation of the sum in Eq. (27) is clearly equal to the integral in Eq. (24) for $D=1$. Note that $p_{n}$ represents the occupation probability of state $n$ within the available states from $n$ to infinity but not within all available states, from 1 to infinity. This is because the summation in Eq. (27) starts from $n$, not from unity and it is the same for the integral term at the denominator: it starts from $n-1$, not from 0 . Therefore, ${ }_{n}^{\zeta} D$ reflects the contributions of states from $n$ to infinity to the partition function. If ${ }_{n}^{\zeta} D=0$, then only the state $n$ contributes while all the states equal to or higher than $n$ contribute with equal probability in the case of ${ }_{n}^{\zeta} D=1$. By following the similar way given in Eqs. (21) and (22), the summation in Eq. (27) can be approximated as

$$
\zeta^{\prime}=\sum_{k=n}^{\infty} \exp \left[-(\alpha k)^{2}\right] \approx\left\{\begin{array}{ll}
\zeta_{L}^{\prime}=\frac{\sqrt{\pi}}{2 \alpha}\{1-\operatorname{Erf}[\alpha(n-1)]\}-\frac{\exp \left[-\alpha^{2}(n-1)^{2}\right]}{2}, & \alpha \leqslant \alpha_{*} \\
\zeta_{H}^{\prime}=\exp \left[-(\alpha n)^{2}\right]+\exp \left[-\alpha^{2}(n+1)^{2}\right], & \alpha>\alpha_{*}
\end{array},\right.
$$

where $\alpha_{*}$ can be determined by the simple numerical solution of $\zeta_{H}^{\prime}=\zeta_{L}^{\prime}$ or $\left|\zeta^{\prime}-\zeta_{L}^{\prime}\right|=\left|\zeta^{\prime}-\zeta_{H}^{\prime}\right|$. Therefore, the analytical approach for the dimension of the $n$th order representation, Eq. (27), is given as

$$
\zeta_{n a} D(\alpha)=\left\{\begin{array}{ll}
1-\frac{\ln \left[1-\frac{\alpha}{\sqrt{\pi}} \frac{\exp \left[-\alpha^{2}(n-1)^{2}\right]}{1-\operatorname{Erf}[\alpha(n-1)]}\right]}{\ln \left[\frac{\alpha}{\sqrt{\pi}} \frac{1-\operatorname{Errf}[\alpha(n-1)]}{-(\alpha n)^{2}},\right.} & \alpha \leqslant \alpha_{*} . \\
\frac{\ln \left\{1+\exp \left[-\alpha^{2}(2 n+1)\right]\right\}}{(\alpha n)^{2}-\ln \left[\frac{\alpha}{\sqrt{\pi}} \frac{2}{1-\operatorname{Er}[\alpha(n-1)]},\right.} & \alpha>\alpha_{*} .
\end{array} .\right.
$$

The accuracies of different orders FIR are compared in Fig. 4(a) for the partition function. It is clearly seen that the maximum error decreases and the error distribution becomes narrower when a higher-order representation is chosen. It seems that the maximum error of second order representation is even less than $2 \%$. Similarly, in Fig. 4(b), variations of ${ }_{n}^{5} D$ are shown for the different order of representations. The characteristic confinement value $\alpha_{c}$, which corresponds to the dimension of $1 / 2(D=1 / 2)$, shifts to the lower confinement values with increasing order. This is an expected result since 

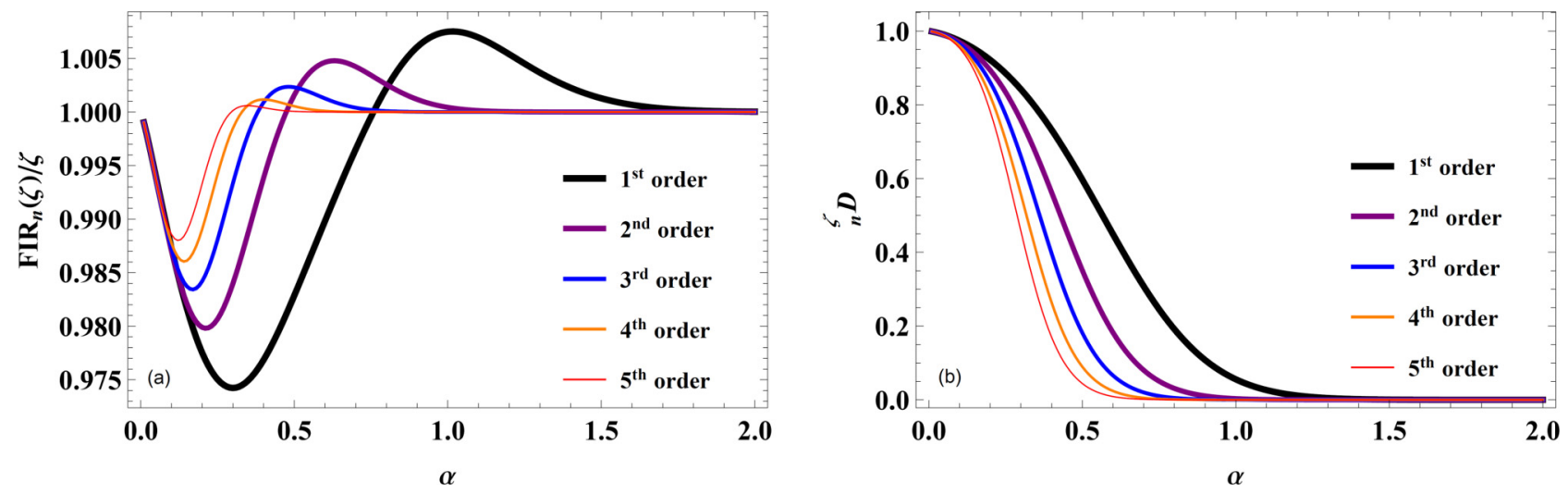

FIG. 4. Accuracies of different orders FIR for the partition function $\operatorname{FIR}_{n}(\zeta) / \zeta$ (a). Variations of dimension for different FIR orders ${ }_{n}^{\zeta} D$ with confinement (b). The thickness of curves decreases with increasing order of FIR for grayscale prints.

the contributions from higher states decay more rapidly when confinement increases.

Consequently, by choosing the higher-order representations $(n \geqslant 2)$, the more accurate results can be obtained while the more individual state terms appear in the resulting expression due to the summation term in Eq. (24). These individual contributions may reduce the compactness of $\operatorname{FIR}(\zeta)$. Moreover, $\operatorname{FIR}_{n}(\zeta)$ has semianalytical solutions, Eq. (26c), for $n \geqslant$ 2 while it has fully analytical and also sufficiently accurate results for $n=1$. However, as mentioned in Sec. IIIC, the second order representation is the minimum order to calculate all thermodynamic properties directly from their fundamental expressions. Therefore, the second-order FIR is chosen also for the partition function here, $\operatorname{FIR}_{2}(\zeta)$, to keep the consistency of the representation orders for direct and indirect calculations of thermodynamic properties. The second order FIR for the partition function is obtained as

$$
\operatorname{FIR}_{2}(\zeta)=\exp \left(-\alpha^{2}\right)+\int_{1}^{\infty} \exp \left[-(\alpha k)^{2}\right] \delta_{D}(k-2) d k
$$

Here the integral term represents the contribution of excited states. When confinement goes to higher values, only the excited states near the first excited state (state 2) contribute to the integral. In the case of ${ }_{2}^{\zeta} D \rightarrow 0$, the only state 2 contributes to the integral since $\delta_{D}(k-2) \rightarrow \delta(k-2)$ and the partition function consists of just the contributions of ground and the first excited states. By use of Eq. (26c), the following semianalytical expression is obtained for Eq. (30):

$$
\begin{aligned}
\operatorname{FIR}_{2}(\zeta)= & \exp \left(-\alpha^{2}\right)+2^{2 D-1}{ }_{2} \mathrm{~F}_{2}\left[\left(\frac{1}{2}, 1\right)\right. \\
& \left.\times\left(\frac{1+D}{2}, \frac{2+D}{2}\right),-(2 \alpha)^{2}\right] \\
& +\frac{\exp \left[-(2 \alpha)^{2}\right]}{2 \alpha^{D}} \Gamma(1+D) \mathrm{FU}\left[\frac{D}{2}, \frac{1}{2},(2 \alpha)^{2}\right] \\
& -{ }_{0}^{1} \Im_{2}^{D} \exp \left[-(\alpha k)^{2}\right] .
\end{aligned}
$$

An analytical expression of the dimension for second order representation is easily obtained from Eqs. (28) and (29) in the following form:

$$
{ }_{2 a}^{\zeta} D(\alpha)=\left\{\begin{array}{ll}
\left.1-\frac{\ln \left[1-\frac{\alpha}{\sqrt{\pi}} \exp \left(-\alpha^{2}\right)\right.}{1-\operatorname{Erf}(\alpha)}\right] & \alpha \leqslant \alpha_{*}=0.475 \\
\frac{\ln \left[\frac{\alpha}{\sqrt{\pi}} \frac{2}{1-\operatorname{Erf}(\alpha)}\right]-(2 \alpha)^{2}}{(2 \alpha)^{2}-\ln \left[\frac{\alpha}{2} \frac{\alpha}{\sqrt{\pi}} \frac{2}{1-\operatorname{Erf}(\alpha)}\right]}, & \alpha>\alpha_{*}=0.475
\end{array} .\right.
$$

The maximum difference between the analytical expression, Eq. (32), and its exact form, Eq. (27), for $n=2$ occurs at $\alpha=\alpha_{*}=0.475$ as 0.06 which causes around $2 \%$ maximum relative difference in Eq. (31).

\section{FIR OF THERMODYNAMIC PROPERTIES}

\section{A. FIR 2 based expressions for the whole range of confinement and dimension}

FIR based expressions can be used to characterize the thermodynamic behavior continuously in the full range of confinement, in which the system undergoes a strong dimensional transition, such as from three dimensions to one dimension. The fundamental expressions of thermodynamic properties are considered in their dimensionless forms and $\mathrm{FIR}_{2}$ is used for the calculations of infinite summations in these expressions. By using the generalized function in Eq. (A3) together with Eqs. (A4) and (A5) for $n=2$, it is possible to represent the dimensionless thermodynamic properties quite easily and successfully. The generalized notation of $\operatorname{FIR}_{2}\left(\alpha, n_{1}, n_{2}\right)$ is used for the simplicity of representations. In this notation, $\operatorname{FIR}_{2}(\zeta)$ in Eq. (31) is represented by $\operatorname{FIR}_{2}(\alpha, 0,2)$. The dimension is calculated by Eq. (A8) for a given set of $\left\{n_{1}, n_{2}, n=2\right\}$. Equations (A5) and (A8) reduce to Eqs. (31) and (32) respectively in the case of $\left\{n_{1}=0, n_{2}=2\right\}$.

FIR expressions of the chemical potential, internal energy, entropy, and heat capacity at constant volume are obtained for both linear and quadratic dispersion relations while their variations due to changes in the confinement are examined only for the quadratic case since there is no qualitative difference between the cases. The only exception is the heat capacity and its different behavior for different dispersions is discussed. 
The dimensionless chemical potential can easily be split into two parts:

$$
\Lambda=\frac{\mu}{k_{b} T}=\ln \left(\frac{N}{\zeta_{t}}\right)=\ln \left(\frac{N}{\zeta_{t, c l}}\right)+\left(\frac{\zeta_{t, c l}}{\zeta_{t}}\right)=\Lambda_{c l}+\Lambda_{e}
$$

where $\Lambda_{c l}=\ln \left(N / \zeta_{t, c l}\right)$ is the classical chemical potential, $\zeta_{t, c l}$ is the classical total partition function given by $\zeta_{t, c l}=$ $\pi^{3 / 2} /\left(8 \alpha_{1} \alpha_{2} \alpha_{3}\right)$, and $\Lambda_{e}$ is the excess chemical potential due to the confinement. Note that $\Lambda_{e}$ goes to zero in the limit of unconfined, i.e., infinitely large, systems in all directions. During the calculations and examinations of FIR for thermodynamic properties, only the excess quantities are considered since the classical terms are trivial and already well known, even in analytical forms. Furthermore, the classical terms are not affected by the changes of confinement or dimension since they are just functions of temperature and density. Therefore, it is always possible to keep the classical terms constant during a change of confinement or dimension. Note that we consider an elongated rectangular box as a confinement domain in which the confinement in the longitudinal direction is negligible $\left(\alpha_{1} \ll 1\right)$ since we use MB statistics, as explained at the end of Sec. II. Therefore, by considering Eq. (33) as well as the condition $\alpha_{1} \ll 1$, which means $\zeta_{1}=\zeta_{1, c l}=\sqrt{\pi} /\left(2 \alpha_{1}\right)$, the excess chemical potential is written as

$$
\begin{aligned}
\Lambda_{e} & =\left(\frac{\zeta_{t, c l}}{\zeta_{t}}\right) \\
& =\ln \left[\frac{\zeta_{2, c l}}{\operatorname{FIR}\left(\alpha_{2}, 0, n_{2}\right)}\right]+\ln \left[\frac{\zeta_{3, c l}}{\operatorname{FIR}\left(\alpha_{3}, 0, n_{2}\right)}\right] \\
& =\Lambda_{2, e}+\Lambda_{3, e}
\end{aligned}
$$

where $\zeta_{2, c l}=\sqrt{\pi} /\left(2 \alpha_{2}\right)$ and $\zeta_{3, c l}=\sqrt{\pi} /\left(2 \alpha_{3}\right)$ are the components of the classical partition function in directions 2 and 3, respectively. Equation (34) represents the correction for $\Lambda_{c l}$ because of the confinement. This correction is not valid only for the limited values of confinements, $\left\{\alpha_{2}, \alpha_{3}\right\} \ll 1$ or $\left\{\alpha_{2}, \alpha_{3}\right\} \gg 1$, but for their whole range, $0<\left\{\alpha_{2}, \alpha_{3}\right\}<\infty$. In other words, we do not need two different or piecewise expressions, one for weak and one for strong confinements, but instead, we have a unified representation due to the dimension controlled nature of $\mathrm{FIR}_{2}\left(\alpha, 0, n_{2}\right)$. When Eq. (34) is added to $\Lambda_{c l}, \zeta_{2, c l}$ and $\zeta_{3, c l}$ terms in Eq. (34) cancel the same terms in

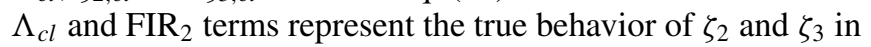
the full range of confinement.

The dimensionless internal energy, $\tilde{u}=U / N k_{b} T$, can also be decomposed for each direction and expressed as

$$
\begin{aligned}
\tilde{u}= & \frac{1}{n_{2}}+\frac{\sum_{k_{2}=1}^{\infty}\left(\alpha_{2} k_{2}\right)^{n_{2}} \exp \left[-\left(\alpha_{2} k_{2}\right)^{n_{2}}\right]}{\sum_{k_{2}=1}^{\infty} \exp \left[-\left(\alpha_{2} k_{2}\right)^{n_{2}}\right]} \\
& +\frac{\sum_{k_{3}=1}^{\infty}\left(\alpha_{3} k_{3}\right)^{n_{2}} \exp \left[-\left(\alpha_{3} k_{3}\right)^{n_{2}}\right]}{\sum_{k_{3}=1}^{\infty} \exp \left[-\left(\alpha_{3} k_{3}\right)^{n_{2}}\right]} .
\end{aligned}
$$

The first term, $1 / n_{2}$, is a result of the first order integral approaches for the summations over $k_{1}$ due to $\alpha_{1} \ll 1$ which allows a continuum approach in that direction. In a similar way to Eq. (34), the excess internal energy is expressed by

$$
\tilde{u}_{e}=\tilde{u}-\frac{3}{n_{2}}=\frac{\operatorname{FIR}_{2}\left(\alpha_{2}, n_{2}, n_{2}\right)}{\operatorname{FIR}_{2}\left(\alpha_{2}, 0, n_{2}\right)}+\frac{\operatorname{FIR}_{2}\left(\alpha_{3}, n_{2}, n_{2}\right)}{\operatorname{FIR}_{2}\left(\alpha_{3}, 0, n_{2}\right)}-\frac{2}{n_{2}} .
$$

Note that since $\tilde{u}$ is a quantity per particle, $\tilde{u}$ and $\tilde{u}_{e}$ consist of the ratios of two summations with different order of moments in Eq. (35) and similarly two different FIR 2 functions in Eq. (36) respectively. The terms in the numerator and denominator have different dimensions. In Appendix A, these dimensions can clearly be seen in Fig. 9 for the curves of $n_{1}=n_{2}$ and $n_{1}=0$ respectively. Therefore, unlike the chemical potential, the dimension of $\tilde{u}_{e}$ is a combination of these dimensions. On the other hand, the dimension of the partition function is the essential one for all thermodynamic quantities since the partition function is a fundamental quantity from which all thermodynamic properties can be derived. Thus, its dimension is chosen here as a reference dimension for the system during the examinations of thermodynamic quantities.

Dimensionless entropy is simply expressed by $\tilde{s}=$ $S / N k_{b}=\tilde{u}-\Lambda+1$ in MB statistics. By subtracting the classical part $\tilde{s}_{c l}=\tilde{u}_{c l}-\Lambda_{c l}+1$, the dimensionless excess entropy, $\tilde{s}_{e}=\tilde{u}_{e}-\Lambda_{e}$, can easily be written by Eqs. (34) and (36) as

$$
\begin{aligned}
\tilde{s}_{e}= & \frac{\operatorname{FIR}_{2}\left(\alpha_{2}, n_{2}, n_{2}\right)}{\operatorname{FIR}_{2}\left(\alpha_{2}, 0, n_{2}\right)}-\ln \left[\frac{\zeta_{2, c l}}{\operatorname{FIR}\left(\alpha_{2}, 0, n_{2}\right)}\right] \\
& +\frac{\operatorname{FIR}_{2}\left(\alpha_{3}, n_{2}, n_{2}\right)}{\operatorname{FIR}_{2}\left(\alpha_{3}, 0, n_{2}\right)}-\ln \left[\frac{\zeta_{3, c l}}{\operatorname{FIR}\left(\alpha_{3}, 0, n_{2}\right)}\right]-\frac{2}{n_{2}} .
\end{aligned}
$$

Finally, the dimensionless specific heat capacity at constant volume, $\tilde{c}_{V}=C_{V} / N k_{b}$, is

$$
\begin{aligned}
\tilde{c}_{V}= & \sum_{r=1}^{3}\left\{\frac{\sum_{k_{r}=1}^{\infty}\left(\alpha_{r} k_{r}\right)^{2 n_{2}} \exp \left[-\left(\alpha_{r} k_{r}\right)^{n_{2}}\right]}{\sum_{k_{r}=1}^{\infty} \exp \left[-\left(\alpha_{r} k_{r}\right)^{n_{2}}\right]}\right. \\
& \left.-\left[\frac{\sum_{k_{r}=1}^{\infty}\left(\alpha_{r} k_{r}\right)^{n_{2}} \exp \left[-\left(\alpha_{r} k_{r}\right)^{n_{2}}\right]}{\sum_{k_{r}=1}^{\infty} \exp \left[-\left(\alpha_{r} k_{r}\right)^{n_{2}}\right]}\right]^{2}\right\} .
\end{aligned}
$$

Because of the condition $\alpha_{1} \ll 1$, the term for $r=1$ becomes simply $1 / n_{2}$ like in Eq. (35) and the excess $\tilde{c}_{V}$ is given by

$$
\begin{aligned}
\tilde{c}_{V}^{e}= & \tilde{c}_{V}-\frac{3}{n_{2}}=\frac{\mathrm{FIR}_{2}\left(\alpha_{2}, 2 n_{2}, n_{2}\right)}{\mathrm{FIR}_{2}\left(\alpha_{2}, 0, n_{2}\right)}-\left[\frac{\mathrm{FIR}_{2}\left(\alpha_{2}, n_{2}, n_{2}\right)}{\operatorname{FIR}_{2}\left(\alpha_{2}, 0, n_{2}\right)}\right]^{2} \\
& +\frac{\mathrm{FIR}_{2}\left(\alpha_{3}, 2 n_{2}, n_{2}\right)}{\mathrm{FIR}_{2}\left(\alpha_{3}, 0, n_{2}\right)}-\left[\frac{\mathrm{FIR}_{2}\left(\alpha_{3}, n_{2}, n_{2}\right)}{\operatorname{FIR}_{2}\left(\alpha_{3}, 0, n_{2}\right)}\right]^{2}-\frac{2}{n_{2}} .
\end{aligned}
$$

Entropy and heat capacity are based on summations containing different orders of moments of energy. Entropy contains the zeroth and first order of moments since it is related to both chemical potential and internal energy. Heat capacity contains the first and second order of moments of energy. Therefore, there is no single and unique dimension to represent their dimensional behaviors; it is a combination of different dimensions for different orders, like in the case of internal energy.

Since the dimension of the partition function is chosen as an essential and reference dimension for the system, the total 


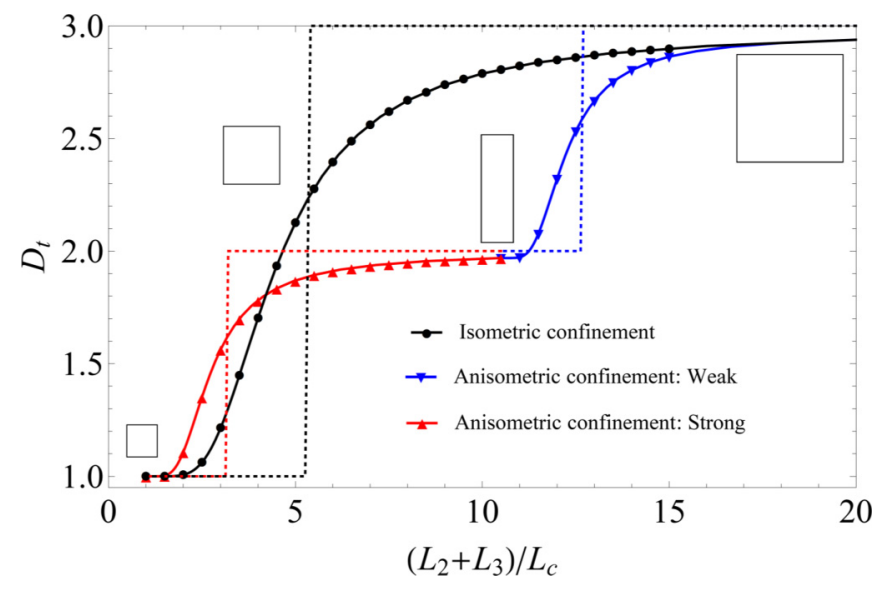

FIG. 5. Variations of dimension for quadratic dispersion relation, $n_{2}=2$, during different peripheral deformation scenarios. Note that $L_{2}+L_{3}$ is half of the periphery in the transverse direction of the confinement domain. The black curve (marked with dots) stands for the isometric changes of confinements in both directions simultaneously, $\alpha_{2}=\alpha_{3}=\{0.1, \ldots, 2\}$. The blue curve (marked with down triangles) represents the weak anisometric confinement scenario in which the only one of the confinements changes $\alpha_{3}=\{0.1, \ldots, 2\}$ while the other one is kept constant at its lowest value, $\alpha_{2}=0.1$. The red curve (marked with up triangles) is for the opposite case: one of the confinements takes its highest value $\alpha_{3}=2$ while the other one changes, $\alpha_{2}=\{0.1, \ldots, 2\}$. The dotted lines show the best possible representations when only integer dimensional approaches are used. Squares and rectangle boxes represent the shape of the cross section of the confinement domain in the transverse direction.

dimension is given in second order representation by

$$
D_{t}=1+{ }_{2}^{\zeta} D\left(\alpha_{2}\right)+{ }_{2}^{\zeta} D\left(\alpha_{3}\right) .
$$

Note that the dimension for direction 1 is always equal to unity due to the condition of $\alpha_{1} \ll 1$.

\section{B. Behaviors of excess thermodynamic properties during the dimensional transitions}

For the examinations here, only the quadratic dispersion relation, $n_{2}=2$, is considered since the results for linear and quadratic dispersion relations are qualitatively very similar to each other as mentioned before. Changes in excess thermodynamic properties are analyzed for different deformation scenarios of confinements in transverse directions. Variations of dimension for these scenarios are given in Fig. 5. There are two anisometric confinement scenarios: weak and strong. The weak one is performed by keeping one of the transverse sizes in its longest value (lowest confinement), $L_{2}=10 L_{c}$ while the other size changes in between its longest and shortest values, $L_{3}=(10, \ldots, 0.5) L_{c}$. The strong one is the opposite scenario, $L_{3}=0.5 L_{c}$ and $L_{2}=(10, \ldots, 0.5) L_{c}$, subsequently following the weak confinement scenario. During the isometric deformation of confinement, both $L_{2}$ and $L_{3}$ simultaneously change in the same interval. In other words, the shape of the domain remains always as a square in isometric deformation. For anisometric scenarios, it is seen that variations of dimension are sharper and stepwise in comparison with that of isometric one. The midpoints of transitions from $3 \mathrm{D}$ to $2 \mathrm{D}$ and from
$2 \mathrm{D}$ to $1 \mathrm{D}$ approximately correspond to the lengths $L_{3} \approx 2.5 L_{c}$ and $L_{2} \approx 2.5 L_{c}$ respectively. This is why the total dimension of the system becomes 2.5 and 1.5 around $L_{2}+L_{3} \approx 12.5 L_{c}$ and $L_{2}+L_{3} \approx 2.5 L_{c}$ correspondingly. In an isometric case, there is a single transition from $3 \mathrm{D}$ to $1 \mathrm{D}$ nearby $L_{2}+L_{3} \approx$ $5 L_{c}$ where $D=2$. Isometric dimensional variation crosses the anisometric one close to $L_{2}+L_{3} \approx 4 L_{c}$. The same scenarios can also roughly be represented by integer dimensional approaches, seen as dotted lines in Fig. 5.

Excess thermodynamic properties are analyzed for these three scenarios. In Fig. 6, variations of excess chemical potential and internal energy are shown. The solid curves represent the true predictions based on direct calculations of infinite summations for the related quantities while the dashed curves (almost match the solid ones) represent the predictions of FIR. It is seen that they are in quite good agreement and the dashed and solid curves almost perfectly match each other. Therefore, FIR of thermodynamic properties provides the unified expressions valid for both weakly and strongly confined cases. On the other hand, the integer dimensional approaches can make only rough predictions, indicated by arrows in Fig. 6, and considerably deviates from the true behaviors especially around the transition regions from one integer representation to another, namely $3 \mathrm{D} \leftrightarrow 2 \mathrm{D}, 2 \mathrm{D} \leftrightarrow 1 \mathrm{D}$, and $3 \mathrm{D} \leftrightarrow 1 \mathrm{D}$. Besides its continuous representation success, FIR also allows establishing dimensional relations between the behavior of a property and the dimension of the partition function under different confinement scenarios. As expected, the excess chemical potential and internal energy increase with reducing sizes (or dimension Fig. 5) due to growing confinement energy. The isometric path gives smooth changes whereas anisometric paths exhibit stepwise behavior both in properties and dimension because of the sequential confinement procedures in different directions of the rectangular domain. Note that in a circular domain, for example, an anisometric path is clearly not possible and only the isometric change of dimension, from three dimensions to one dimension, can be realized. Therefore, different shapes allow different scenarios for dimensional transitions.

The regions in between the black curve and the blue and red curves represent all available thermodynamic states for the considered interval of confinement parameters, (0.1-2). Therefore, these figures also show the possibility of novel thermodynamic cycles based on dimensional changes in momentum space. If we consider internal energy as an example, we can change the confinement by following the black path from $3 \mathrm{D}$ to $1 \mathrm{D}$ and then turned back to the initial state by following the red and blue curves respectively. In this way, we can generate energy from this cycle. Note that change of confinements, $\alpha_{2}, \alpha_{3}$, can be realized either by an isothermal change in sizes or isometric changes in temperature. Therefore, not only work exchange but also heat exchange processes can be performed through this cycle.

In Fig. 7, it is seen that excess entropy takes negative values with increasing confinement up to a critical value and then becomes positive while the excess heat capacity exhibits the opposite behavior. Note that the arrows here indicate the curves based on integer dimensional approaches, which match the true behavior only in a very limited interval of confinement (peripheral length) and cannot predict the negative values of 

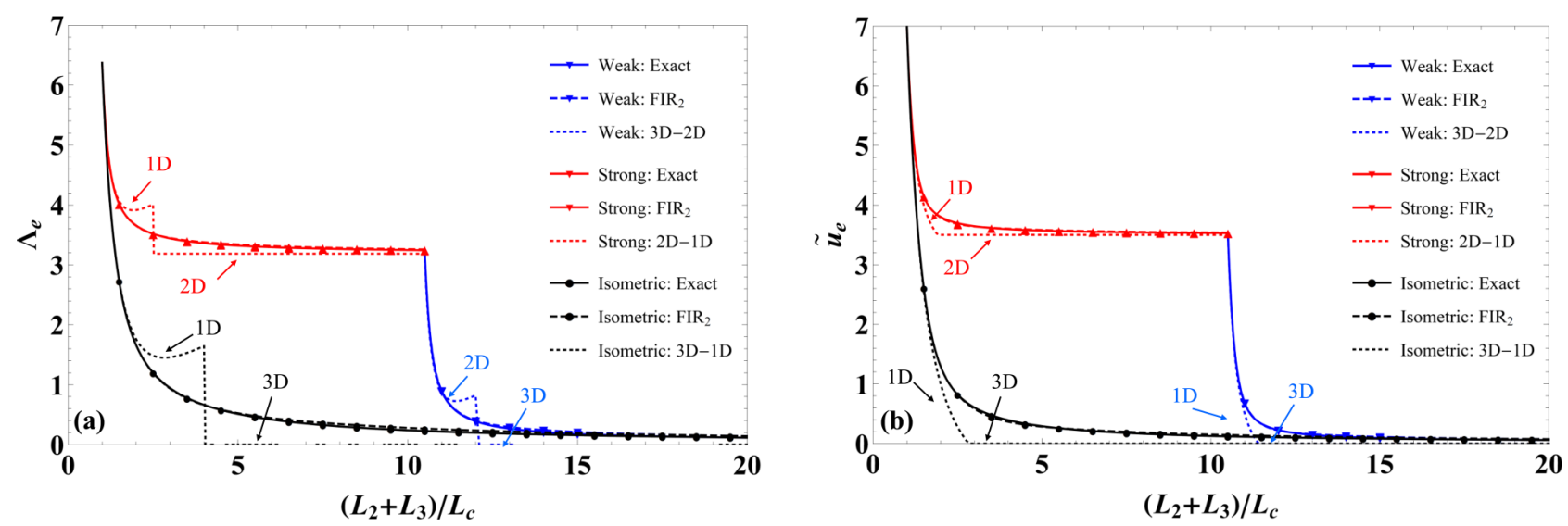

FIG. 6. Excess chemical potential (a) and excess internal energy (b) vs different peripheral deformation scenarios. Dashed curves (almost match on the solid ones) represent FIR results while the solid ones are the exact ones. The dispersion relation is quadratic, $n_{2}=2$. The dotted curves show the best possible representations when only integer dimensional approaches are used. Arrows show the dimension of the approaches for the best match.

excess entropy. This behavior in entropy can be explained by its mathematical relations with the internal and chemical potential energies, $\tilde{s}_{e}=\tilde{u}_{e}-\Lambda_{e}$. If we look at the behaviors of $\tilde{u}_{e}$ and $\Lambda_{e}$ in Fig. 6, both of them monotonically increase with increasing confinement (decreasing periphery). However, the increment in $\tilde{u}_{e}$ becomes slightly sharper and dominant in comparison with that of $\Lambda_{e}$ if the confinement goes beyond a certain point. This results in positive excess entropy. If we calculate the excess entropy when $\left\{\alpha_{3}>1, \alpha_{2} \ll 1\right\}$ which corresponds to the conditions where the blue curve of excess entropy crosses the zero axes, we get $\tilde{s}_{e} \rightarrow-\frac{1}{2}-\ln \left(\frac{\sqrt{\pi}}{2 \alpha_{3}}\right)$. It turns into a positive sign when $\alpha_{3}>1.46$ which corresponds to $L_{3} / L_{c}=1 / 1.46 \approx 0.7$. Since through the blue curve $L_{2} / L_{c}=10,\left(L_{2}+L_{3}\right) / L_{c}=10.7$ which is the zerocrossing point of the blue curve. Similar calculations can be done for $\left\{\alpha_{3}>1, \alpha_{2}>1\right\}$ and we get $\alpha_{2} \alpha_{3}=2.1$. When the confinements are equal to each other, it corresponds to $\left(L_{2}+L_{3}\right) / L_{c}=1.4$ which is the zero-crossing point for the black curve of excess entropy. These simple mathematical explanations show that the positive excess entropy occurs to eliminate the incorrect contributions of the classical partition function in the classical entropy expression. The terms $-\ln \left(\zeta_{2, c l}\right)$ and $-\ln \left(\zeta_{3, c l}\right)$ in Eq. (37) are eliminated by the same terms with opposite sign in the classical entropy expression. Therefore, when we examine the entropy, instead of the excess one, we see that it always decreases with growing confinement, never increases, in an agreement with our expectations.

Excess heat capacity has positive contributions if the confinement is weak enough for at least one direction. Positive contribution causes overheat capacity, which cannot be predicted by integer dimensional representations. For strong confinement conditions, the degree of freedom for translational motion is lost and heat capacity decreases the amount of $1 / 2$ for each confined direction. As it is well known and seen in Eq. (38), the heat capacity is the variance of the energy,
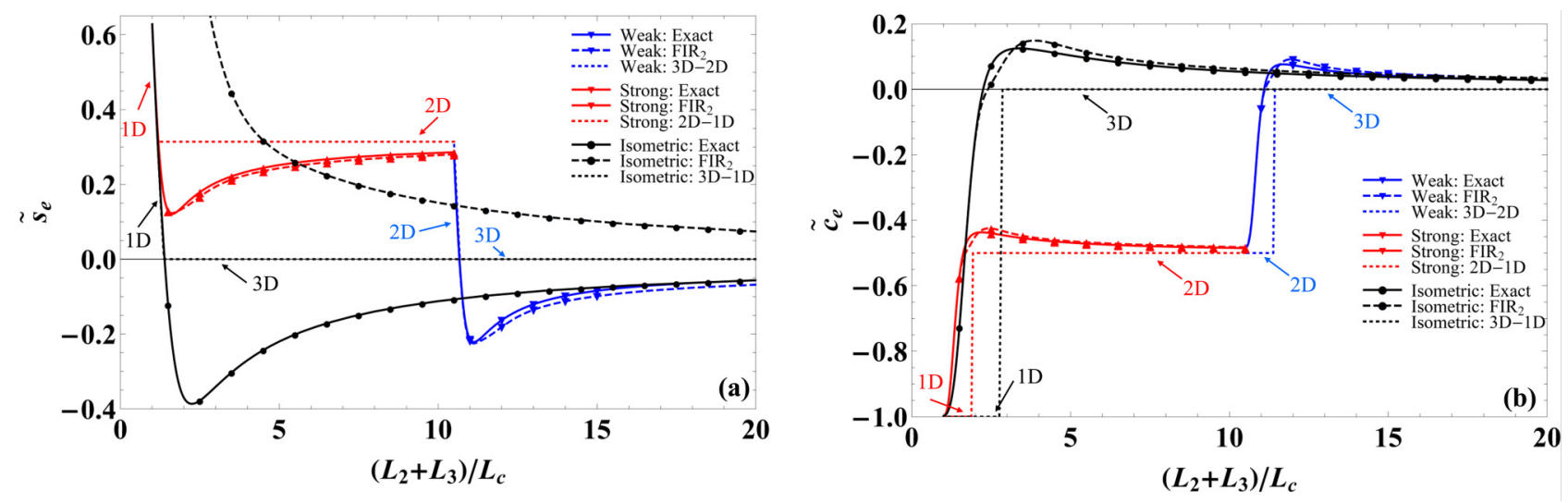

FIG. 7. Excess entropy (a) and excess heat capacity (b) vs different peripheral deformation scenarios. Dashed curves (almost match on the solid ones) represent FIR results while the solid ones are the exact ones. The dispersion relation is quadratic here, $n_{2}=2$. The dotted curves show the best possible representations when only integer dimensional approaches are used. Arrows show the dimension of the approaches for the best match. 
$\tilde{c}_{V}=\overline{\tilde{\varepsilon}^{2}}-\overline{\tilde{\varepsilon}}^{2}$. In the case of quadratic dispersion, $n_{2}=2, \overline{\tilde{\varepsilon}^{2}}$ and $\overline{\tilde{\varepsilon}}^{2}$ approach $3 / 4$ and $1 / 4$, respectively, as the confinement tends to zero, which leads to the classical $1 / 2$ value of heat capacity for each free direction. Therefore, when $\left(L_{2}+L_{3}\right) / L_{c} \gg 1$, the excess heat capacity goes to zero. In the strongly confined limit, both of them go to $\alpha^{4}$ and it gives zero value for each confined direction. This causes the value of -1 for the excess heat capacity in the limit of $\left(L_{2}+L_{3}\right) / L_{c} \rightarrow 0$ to cancel the classical $1 / 2+1 / 2$ terms for the directions of 2 and 3. On the other hand, when confinement increases, $\overline{\tilde{\varepsilon}^{2}}$ goes to $\alpha^{4}$ faster than $\overline{\tilde{\varepsilon}}^{2}$ and this behavior causes positive values in excess heat capacity. When the second one catches the first one, their difference becomes zero and $\tilde{c}_{V}^{e}$ equals minus unity. Although the results of linear and quadratic cases are very similar for most of the thermodynamic quantities considered here, the situation is different for heat capacity. In the case of the linear dispersion relation, the positive contribution of confinement disappears and $\tilde{c}_{V}^{e}$ takes always negative values, $\tilde{c}_{V}^{e}<0$. In other words, there is no overheat capacity in the case of linear dispersion.

\section{CONCLUSION}

The FIR is proposed for calculations of the partition function as well as for the common infinite sums appearing in statistical thermodynamics. The dimension of an infinite sum for a scaled function is introduced as a control parameter of the FIR. By using the proposed method, it is seen that we are able to express the thermodynamic properties of confined systems directly as a function of the dimension of momentum space. Because of the FIR and the dimension defined in this study, thermodynamic quantities can be examined not only for integer dimensions but also for fractional ones. These expressions are unified expressions valid for the full range of confinement and dimension, instead of their limited ranges. The proposed method can be used when the momentum eigenvalues of particles are explicitly known. FIR is applied for a generic function representing the most common functions in statistical thermodynamics and excess thermodynamic quantities are analyzed during the continuous change in the wide range of confinement. It seems that the FIR can successfully predict the true behavior of thermodynamic quantities. Application of FIR is also extended to the widely used quadratic and quartic confinement potentials besides the infinite well, and the success of FIR is shown for these potentials in Appendix B. FIR seems to be a powerful method to calculate the various infinite sums in statistical physics. This opens up some applications of fractional integrals in statistical physics as well. The applications can be extended to the thermodynamics of Fermi and Bose gases. Especially, it may be interesting to consider Bose-Einstein condensation by the proposed method, FIR, since it directly relates the thermodynamic behaviors with dimension and allows us to examine quantities during dimensional transitions. Not only thermodynamic properties but also transport properties can be calculated by the FIR since their fundamental expressions are also based on infinite sums. Thus, many interesting phenomena based on confinement effects can be investigated by the FIR. Furthermore, it constitutes a base for the derivation of DOS for arbitrary dimensions, including fractional ones. Moreover, it seems that dimension can serve as a state function in thermodynamics and results in numerous new processes and thermodynamic cycles, especially at the nanoscale. These topics are now under consideration as extensions of the study by the same group.

\section{ACKNOWLEDGMENTS}

A.S. would like to express his deep gratitude to the Material Theory Division of Astronomy \& Physics Department of Uppsala University for their kind and warm hospitality. J.F. acknowledges the support from Vetenkapsrådet.

\section{APPENDIX A: FIR FOR WIDELY USED SUMMATIONS IN STATISTICAL THERMODYNAMICS}

During the calculations of thermodynamic and transport properties, not only the partition function but also the summations consisting of the distribution function multiplied by the powers of momentum, energy, and even some other functions need to be calculated. For example, calculation of the ensemble sum of energy, $\sum \tilde{\varepsilon} \exp (-\tilde{\varepsilon})=\sum(\alpha k)^{2} \exp \left[-(\alpha k)^{2}\right]$, is necessary to directly calculate the internal energy. Similarly, the ensemble sum of energy square, $\sum \tilde{\varepsilon}^{2} \exp (-\tilde{\varepsilon})=\sum(\alpha k)^{4} \exp \left[-(\alpha k)^{2}\right]$, is needed for the direct calculation of heat capacity. Besides, the dispersion relation may not always be quadratic but it may be linear. Therefore, instead of considering only the partition function and only the quadratic energy-momentum relation, there is a need to generalize FIR for a generic function representing the most common functions in statistical thermodynamics. Equation (26a) can be conjectured to be valid for a convergent infinite sum over $k$ from one to infinity, $A(\alpha)$, of a non-negative function scaled by $\alpha, f(\alpha k)$, as

$$
\begin{aligned}
A(\alpha)= & \sum_{k=1}^{\infty} f(\alpha k) \Rightarrow \operatorname{FIR}_{n}(A) \\
= & \sum_{k=1}^{n-1} f(\alpha k)+\int_{0}^{\infty} f(\alpha k) \delta_{D}(k-n) d k \\
& -\int_{0}^{n-1} f(\alpha k) \delta_{D}(k-n) d k \\
= & \sum_{k=1}^{n-1} f(\alpha k)+{ }_{0}^{\infty} \Im_{n}^{D} f(\alpha k)-{ }_{0}^{n-1} \Im_{n}^{D} f(\alpha k) .
\end{aligned}
$$

Dimension is defined by considering Eq. (27) and $f(\alpha k)$ as

$$
{ }_{n}^{A} D(\alpha)=\frac{\ln \left[w_{n}(\alpha)\right]}{\ln \left[w_{n, c l}(\alpha)\right]}=\frac{\ln \left[\frac{f(\alpha n)}{\sum_{k=n}^{\infty} f(\alpha n)}\right]}{\ln \left[\frac{f(\alpha n)}{\int_{n-1}^{\infty} f(\alpha n) d k}\right]} .
$$

Note that $w_{n}$ is not the probability anymore but the weight factor of state $n$. When $f(\alpha k)$ becomes a distribution function, then $w_{n}$ becomes the probability of state $n$. Depending on both the nature of $f(\alpha k)$ and the value of $\alpha$, there is a possibility that $w_{n, c l}(\alpha)>1$ while $w_{n}(\alpha)<1$. In this case, dimension takes the unphysical negative values and this situation shows that the selected order of FIR is not sufficient to represent the sum. This can easily be overcome just by selecting the higher 
$n$ values, going to higher-order representations. The second order is the minimum order to calculate the ensemble sum of energy square for example.

For the most common calculations in statistical thermodynamics, a quite useful and generic function can be defined as follows:

$$
f(\alpha k)=(\alpha k)^{n_{1}} \exp \left[-(\alpha k)^{n_{2}}\right]
$$

where $n_{1} \geqslant 0$ and $n_{2}=2$ or 1 depending on if the dispersion relation is quadratic or linear respectively. Note that Fermi and Bose distribution functions can be expended in terms of the Maxwell-Boltzmann distribution function. Therefore, the calculations and the results here constitute a base for FIR in statistical thermodynamics in general. For the generic function in Eq. (A3), the term ${ }_{0}^{\infty} \Im_{n}^{D} f(\alpha k)$ in Eq. (A1b) can even be analytically calculated. For linear dispersion relation, $n_{2}=1$, it is obtained as

$$
\begin{aligned}
\underset{0}{\infty} \Im_{n}^{D} f(\alpha k)= & 2^{-1+D} \alpha^{-D} \frac{D}{D+n_{1}} \Gamma\left(1+D+n_{1}\right)_{1} F_{1}\left(1-D, 1-D-n_{1},-n \alpha\right)+2^{-1+D} \alpha^{n_{1}} n^{n_{1}+D}{ }_{1} F_{1}\left(1+n_{1}, 1+D+n_{1},-n \alpha\right) \\
& \times\left[\frac{\Gamma(1+D) \Gamma\left(1+n_{1}\right)}{\Gamma\left(1+D+n_{1}\right)}-\frac{\Gamma(1+D)}{\Gamma\left(-n_{1}\right)} \frac{\Gamma\left(1-D-n_{1}\right)}{D+n_{1}}\right],
\end{aligned}
$$

where ${ }_{p} F_{q}(z)=F\left[\left(a_{1}, \ldots, a_{p}\right),\left(b_{1}, \ldots, b_{q}\right), z\right]$ is the generalized hypergeometric function. For a quadratic dispersion relation, $n_{2}=2, \underset{0}{\infty} \mathfrak{\Im}_{n}^{D} f(\alpha k)$ is solved as

$$
\begin{aligned}
{ }_{0}^{\infty} \Im_{n}^{D} f(\alpha k)= & 2^{-1+D} \alpha^{-D} \frac{D}{D+n_{1}} \Gamma\left(\frac{2+D+n_{1}}{2}\right){ }_{2} F_{2}\left[\left(\frac{1-D}{2}, \frac{2-D}{2}\right),\left(\frac{1}{2}, \frac{2-D-n_{1}}{2}\right),-(n \alpha)^{2}\right] \\
& +2^{-1+D} \alpha^{1-D} \frac{n D(1-D)}{D+n_{1}-1} \Gamma\left(\frac{1+D+n_{1}}{2}\right){ }_{2} F_{2}\left[\left(\frac{2-D}{2}, \frac{3-D}{2}\right),\left(\frac{3}{2}, \frac{3-D-n_{1}}{2}\right),-(n \alpha)^{2}\right] \\
& +2^{-1+D} \alpha^{n_{1}} n^{n_{1}+D} \Gamma(1+D)\left[\frac{1}{\Gamma\left(1+D+n_{1}\right)}-\frac{\Gamma\left(1-D-n_{1}\right)}{\Gamma\left(-n_{1}\right)\left(D+n_{1}\right)}\right]{ }_{2} F_{2} \\
& \times\left[\left(\frac{1+n_{1}}{2}, \frac{2+n_{1}}{2}\right),\left(\frac{1+D+n_{1}}{2}, \frac{2+D+n_{1}}{2}\right),-(n \alpha)^{2}\right] .
\end{aligned}
$$

Note that since the Gamma function goes to infinity for negative integer numbers, the last terms in Eqs. (A4) and (A5) vanish for the integer number of moment exponent, $n_{1}$. In the case of $n_{1}=0$, Eq. (A5) simply reduces to Eq. (15b) for $n=1$ and to the summation of the second and third terms in Eq. (31) for $n=2$.

Although we get fully analytical results for the first integral in Eq. (A1a), the second integral ${ }_{0}^{n-1} \mathfrak{\Im}_{n}^{D} f(\alpha k)$ should be calculated numerically. Nevertheless, this numerical integration is an easy process since the singularity in the integrand, as a result of $\delta_{D}(k-n)$ when $D \rightarrow 0$, remains out of the integration region. Therefore, the difficult part is already solved out by the analytical integration of the first one, ${ }_{0}^{\infty} \Im_{n}^{D} f(\alpha k)$.

The analytical form of the dimension for Eq. (A2) is obtained by following the same recipe as in Eq. (29). For this purpose, the following quantities are defined:

$$
A^{\prime}=\sum_{k=n}^{\infty} f(\alpha k) \approx\left\{\begin{array}{ll}
A^{\prime}{ }_{L}=\int_{n-1}^{\infty} f(\alpha k) d k-\frac{f[\alpha(n-1)]}{2}, & \alpha \leqslant \alpha_{*} \\
A^{\prime}{ }_{H}=f(\alpha n)+f[\alpha(n+1)], & \alpha>\alpha_{*}
\end{array},\right.
$$

where $\alpha_{*}$ is obtained by the numerical solution of $\left|A^{\prime}-A^{\prime}{ }_{L}\right|=\left|A^{\prime}-A_{H}^{\prime}\right|$. By use of Eq. (A3) in Eq. (A6), we obtain

$$
A^{\prime} \approx \begin{cases}A_{L}^{\prime}=\frac{\Gamma\left[\frac{1+n_{1}}{n_{2}}, \alpha^{n_{2}}(n-1)^{n_{2}}\right]}{\alpha n_{2}}-\frac{\alpha^{n_{1}}(n-1)^{n_{1}} \exp \left[-\alpha^{n_{2}}(n-1)^{n_{2}}\right]}{2}, & \alpha \leqslant \alpha_{*} . \\ A_{H}^{\prime}=(\alpha n)^{n_{1}} \exp \left[-(\alpha n)^{n_{2}}\right]+\alpha^{n_{1}}(n+1)^{n_{1}} \exp \left[-\alpha^{n_{2}}(n+1)^{n_{2}}\right], & \alpha>\alpha_{*}\end{cases}
$$

Therefore, the dimension of the generic function in Eq. (A3) is analytically expressed as

$$
{ }_{n a}^{A} D={ }_{n a}^{n_{1}, n_{2}} D=\frac{\ln \left[\frac{(\alpha n)^{n_{1}} \exp \left[-(\alpha n)^{n_{2}}\right]}{A^{\prime}}\right]}{\ln \left[\frac{\alpha n_{2}(\alpha n)^{n_{1}} \exp \left[-(\alpha n)^{n_{2}}\right]}{\Gamma\left[\frac{1+n_{1}}{n_{2}}, \alpha^{n_{2}}(n-1)^{n_{2}}\right]}\right]}
$$

Equations (A4) and (A5) together with Eqs. (A7) and (A8) provide a generic formulation of FIR for the wide range of

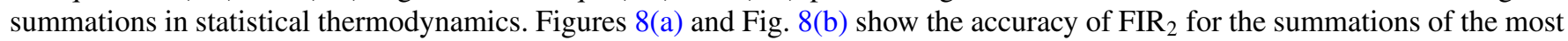
common functions in statistical thermodynamics for quadratic $\left(n_{2}=2\right)$ and linear $\left(n_{2}=1\right)$ dispersion relations respectively. The functions are chosen as the summations of distribution function $\left(n_{1}=0\right)$, energy $\left(n_{1}=n_{2}\right)$, and square of energy $\left(n_{1}=2 n_{2}\right)$. It is seen that the errors of $\mathrm{FIR}_{2}(A)$ are around $2 \%$.

Comparisons of the true dimensions ${ }_{n}^{A} D$, Eq. (A2), with the analytical ones ${ }_{n a}^{A} D$, Eq. (A8), are also given in Figs. 9(a) and 9(b) for quadratic and linear dispersion relations respectively. Maximum differences in between ${ }_{2}^{A} D$ and ${ }_{2 a}^{A} D$ are around 0.07 , 0.08 , and 0.08 for $n_{1}=\left\{0, n_{2}, 2 n_{2}\right\}$ respectively in the case of $n_{2}=2$ while they are $0.15,0.10$, and 0.05 for $n_{2}=1$. It is always 

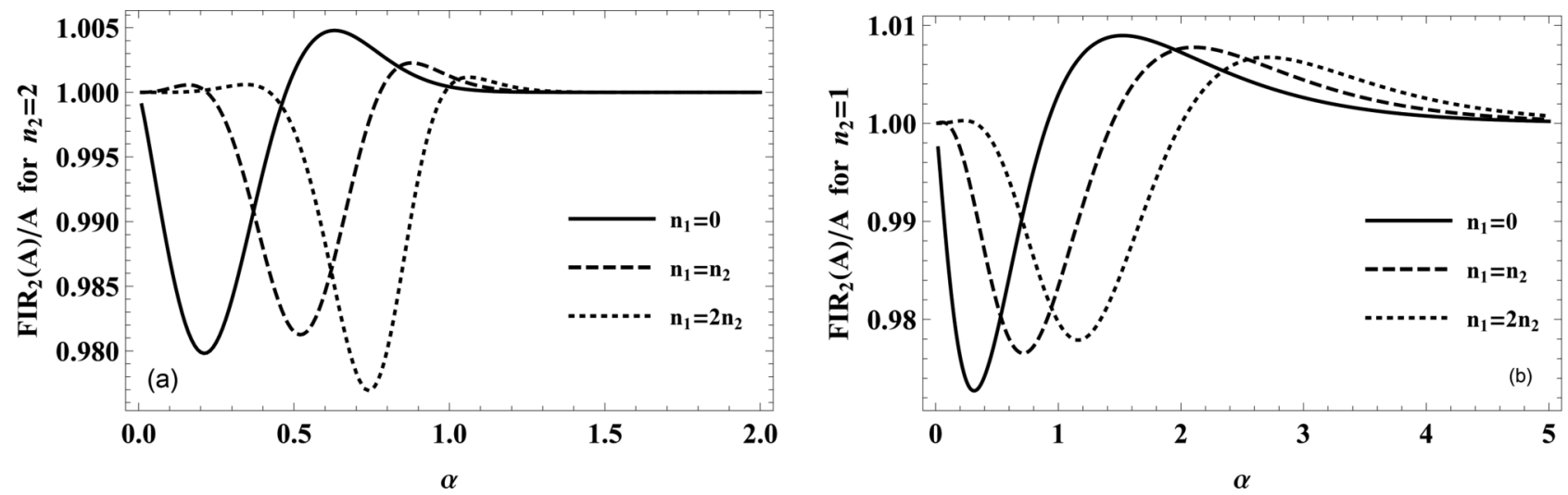

FIG. 8. For quadratic, $n_{2}=2$, (a) and linear, $n_{2}=1$, (b) dispersion relations, $\operatorname{FIR}_{2}(A) / A$ vs confinement for different moment exponent values which correspond to the partition function $\left(n_{1}=0\right)$, energy $\left(n_{1}=n_{2}\right)$, and square of energy $\left(n_{1}=2 n_{2}\right)$.

possible to decrease these errors by considering the first term $(s=1)$ in the third term of PSF, Eq. (4), to calculate the sum in Eq. (A2) more precisely.

\section{APPENDIX B: FIR OF PARTITION FUNCTIONS FOR QUADRATIC AND QUARTIC CONFINEMENT POTENTIALS}

Besides the infinite-well potentials, quadratic and quartic potentials are also widely used for confinement. Therefore, FIR is applied also for the partition functions of particles confined by these potentials. By considering the quadratic and quartic potentials with energy constant $\gamma, U_{2 q}=\gamma x^{2}$ and $U_{4 q}=\gamma x^{4}$ respectively, energy eigenvalues of particles can simply be expressed for each degree of freedom (direction) as follows respectively:

$$
\begin{gathered}
\varepsilon_{q 2}=\hbar \omega_{0, q 2}\left(n+\frac{1}{2}\right), \quad \omega_{0, q 2}=\sqrt{\frac{2 \gamma}{m}} \\
\varepsilon_{q 4}=\left[\hbar \omega_{0, q 4}\left(n+\frac{1}{2}\right)\right]^{4 / 3}, \quad \omega_{0, q 4}=\sqrt{\frac{\pi}{2}} \frac{\Gamma\left(\frac{7}{4}\right)}{\Gamma\left(\frac{5}{4}\right)} \frac{\gamma^{1 / 4}}{\sqrt{m}}=C \frac{\gamma^{1 / 4}}{\sqrt{m}},
\end{gathered}
$$

where $n=0,1,2, \ldots$ is a quantum state variable, $m$ is particle mass, $C \approx 1.2708$, and subscripts of $q 2$ and $q 4$ indicate the quadratic and quartic cases respectively. For the calculations of partition functions, we need to express the quantity of $\varepsilon / k_{b} T$ which can be written in the following forms for quadratic and quartic potentials respectively:

$$
\begin{gathered}
\tilde{\varepsilon}_{q 2}=\frac{\varepsilon_{q 2}}{k_{b} T}=\alpha_{q 2}^{2}\left(n+\frac{1}{2}\right), \quad \alpha_{q 2}=\frac{L_{t h}}{L_{0, q 2}}, \\
\tilde{\varepsilon}_{q 4}=\frac{\varepsilon_{q 4}}{k_{b} T}=\alpha_{q 4}^{2}\left(n+\frac{1}{2}\right)^{4 / 3}, \quad \alpha_{q 4}=\frac{L_{t h}}{L_{0, q 4}} \frac{C}{2^{1 / 3}},
\end{gathered}
$$
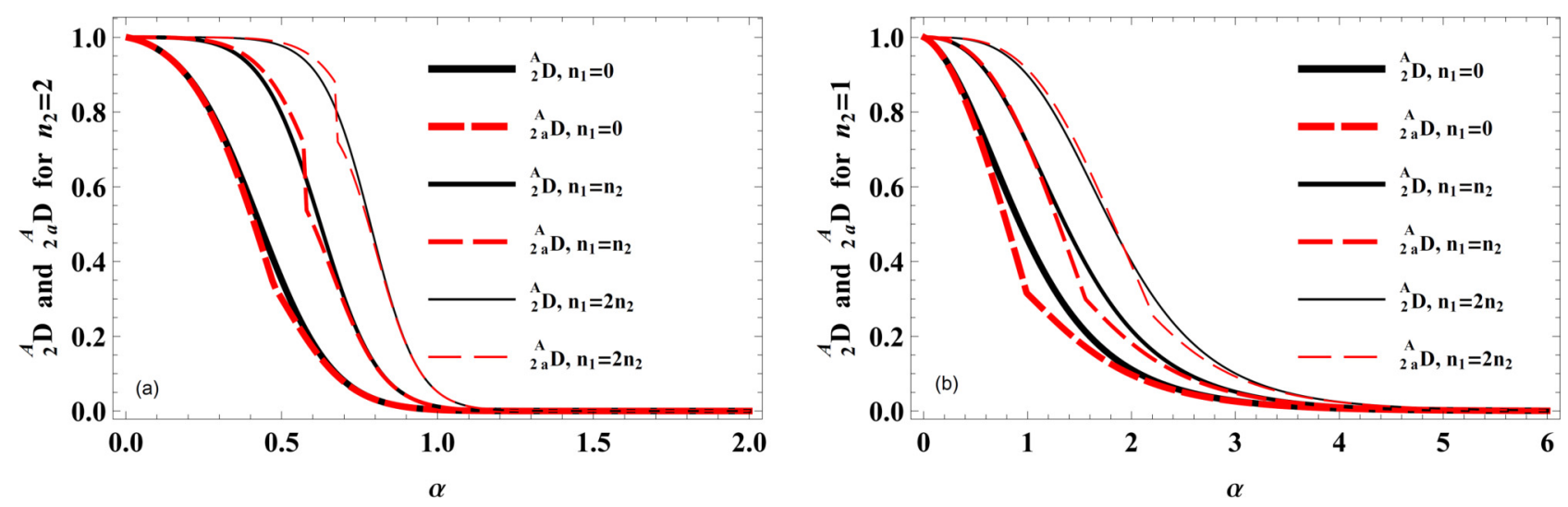

FIG. 9. For quadratic $n_{2}=2$ (a) and linear $n_{2}=1$ (b) dispersion relations, variations of dimension ${ }_{2}^{A} D$ and its approximate analytical form ${ }_{2 a}^{A} D$ with confinement for different moment exponents which correspond to the partition function $\left(n_{1}=0\right)$, energy $\left(n_{1}=n_{2}\right)$, and square of energy $\left(n_{1}=2 n_{2}\right)$. Black (solid) curves represent the true dimension, ${ }_{2}^{A} D$, while the red (dashed) ones stand for analytical ones, ${ }_{2 a}^{A} D$. 

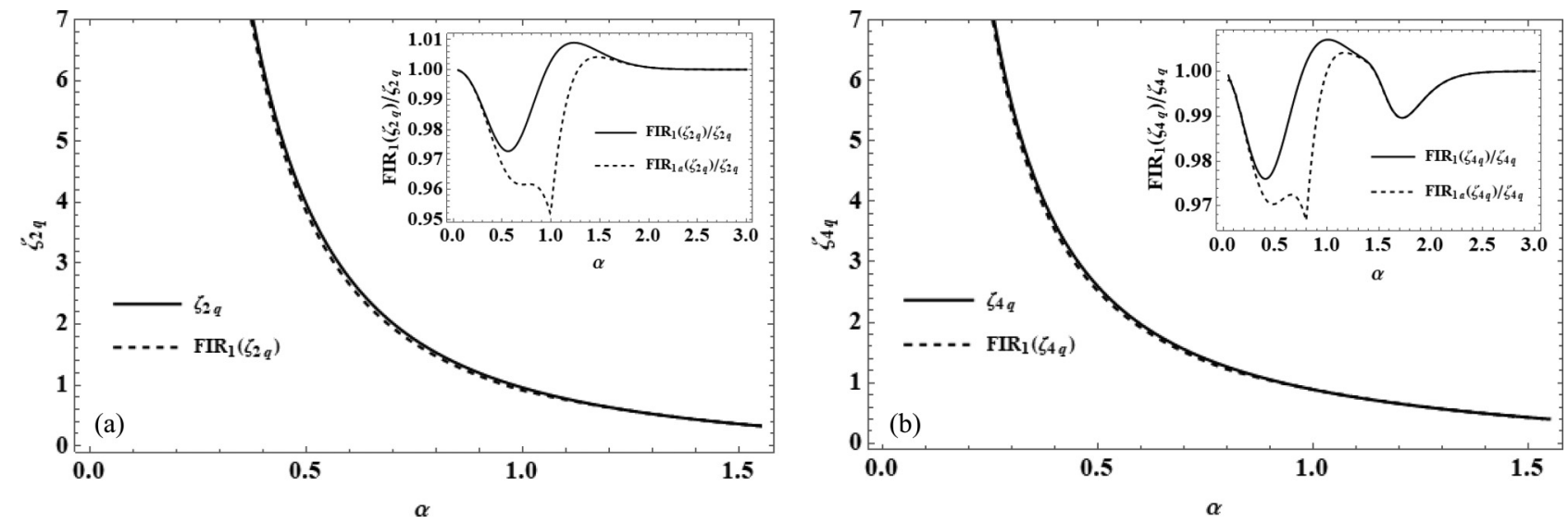

FIG. 10. FIR for partition functions in cases of quadratic (a) and quartic confinement potentials (b). Inset figures show the accuracy distributions of FIR in confinement space when the true and approximate dimension functions are used.

where $\alpha_{q 2}$ and $\alpha_{q 4}$ are the confinement parameters, $L_{0, q 2}=\left(\hbar / m \omega_{0, q 2}\right)^{1 / 2}$ and $L_{0, q 4}=\left(\hbar / m^{3 / 2} \omega_{0, q 4}^{2}\right)^{1 / 3}\left(C / 2^{1 / 3}\right)$ are the minimum displacements corresponding to the zero-point energies $(n=0)$, note that $C / 2^{1 / 3} \approx 1.00865$, and $L_{t h}=\hbar / \sqrt{m k_{b} T}$ is a length scale in the order of the thermal de Broglie wavelength of particles. Therefore, depending on the level of temperature $T, \alpha_{q 2}$ and $\alpha_{q 4}$ can be smaller or bigger than unity. Partition functions can then be expressed for each degree of freedom as

$$
\begin{gathered}
\zeta_{q 2}=\sum_{n=0}^{\infty} \exp \left[-\alpha_{q^{2}}^{2}\left(n+\frac{1}{2}\right)\right]=\exp \left(-\frac{\alpha_{q^{2}}^{2}}{2}\right)+\sum_{n=1}^{\infty} \exp \left[-\alpha_{q 2}^{2}\left(n+\frac{1}{2}\right)\right], \\
\zeta_{q 4}=\sum_{n=0}^{\infty} \exp \left[-\alpha_{q^{4}}^{2}\left(n+\frac{1}{2}\right)^{4 / 3}\right]=\exp \left(-\frac{\alpha_{q^{4}}^{2}}{2^{4 / 3}}\right)+\sum_{n=1}^{\infty} \exp \left[-\alpha_{q^{4}}^{2}\left(n+\frac{1}{2}\right)^{4 / 3}\right] .
\end{gathered}
$$

Note that as it is explained in Sec. III B, the first (zero) term is separated and FIR is applied for the rest of the sum. FIR of $\zeta_{2 q}$ gives even the analytical result as follows:

$$
\begin{gathered}
\operatorname{FIR}_{1}\left(\zeta_{q 2}\right)=\exp \left(-\frac{\alpha_{q^{2}}^{2}}{2}\right)+\int_{0}^{\infty} \exp \left[-\alpha_{q 2}^{2}\left(n+\frac{1}{2}\right)\right] \delta_{D}(n-1) d n, \\
\operatorname{FIR}_{1}\left(\zeta_{q 2}\right)=\exp \left(-\frac{\alpha_{q^{2}}^{2}}{2}\right)+2^{D-1} \alpha_{q 2}^{-2 D} \Gamma(1+D)\left\{1+\exp (-I \pi D)\left[1-\frac{\Gamma\left(D,-\alpha_{q 2}^{2}\right)}{\Gamma(D)}\right]\right\} \exp \left(-\frac{3}{2} \alpha_{q 2}^{2}\right) .
\end{gathered}
$$

The imaginary components appearing in Eq. (B8) due to $\exp (-I \pi D)$ and incomplete Gamma function $\Gamma\left(D,-\alpha_{q 2}^{2}\right)$ cancel each other and Eq. (B8) always results in a real number. FIR of $\zeta_{4 q}$ can be calculated numerically by

$$
\operatorname{FIR}_{1}\left(\zeta_{q^{4}}\right)=\exp \left(-\frac{\alpha_{q^{4}}^{2}}{2^{4 / 3}}\right)+\int_{0}^{\infty} \alpha_{q^{4}}^{2}\left(n+\frac{1}{2}\right)^{4 / 3} \delta_{D}(n-1) d n .
$$

By using a similar way for Eq. (22), approximate analytical expressions for dimensions of $\zeta_{2 q}$ and $\zeta_{4 q}$ can be obtained as follows, respectively:

$$
\begin{aligned}
& { }_{1}^{\zeta_{2 q}} D(\alpha) \approx\left\{\begin{array}{ll}
1-\frac{\ln \left(1-\frac{\alpha^{2}}{2}\right)}{2 \ln \left(\alpha-\alpha^{2}\right.}, & \alpha \leqslant \alpha_{*}=0.996 \\
\frac{\ln \left[1+\exp \left(-\alpha^{2}\right)\right]}{\alpha^{2}-2 \ln (\alpha)}, & \alpha>\alpha_{*}=0.996
\end{array},\right. \\
& { }_{1}^{\zeta 4 q} D(\alpha) \approx\left\{\begin{array}{ll}
1-\frac{\ln \left(1-\frac{2 \alpha^{3 / 2} \exp \left(-\alpha^{2} / 2^{4 / 3}\right)}{3 \Gamma\left[3 / \alpha^{2} / 2^{2 / 3}\right]}\right)}{\ln \left[\frac{4}{3} \alpha^{3 / 2} \exp \left(-\alpha^{2}\left(\frac{3}{2}\right)^{4 / 3}\right) / \Gamma\left[3 / 4, \alpha^{2} / 2^{4 / 3}\right]\right]}, & \alpha \leqslant \alpha_{*}=0.799 \\
-\frac{\ln \left\{1+\exp \left[-\alpha^{2}\left(\left(\frac{5}{2}\right)^{4 / 3}-\left(\frac{3}{2}\right)^{4 / 3}\right)\right]\right\}}{\ln \left[\frac{4}{3} \alpha^{3 / 2} \exp \left(-\alpha^{2}\left(\frac{3}{2}\right)^{4 / 3}\right) / \Gamma\left[3 / 4, \alpha^{2} / 2^{4 / 3}\right]\right]}, & \alpha>\alpha_{*}=0.799
\end{array} .\right.
\end{aligned}
$$

Figures 10(a) and Fig. 10(b) explicitly show the successes of FIR for the calculations of partition functions also in cases of quadratic and quartic confinement potentials. Maximum errors are in the order of $2.5 \%$ when the true dimension functions are used and the errors become $5 \%$ and $3 \%$ for quadratic and quartic potentials respectively if the approximate analytical expressions given by Eqs. (B10) and (B11) are used. FIR seems to be a powerful method to calculate the various infinite sums in statistical physics. 
[1] M. I. Molina, Am. J. Phys. 64, 503 (1996).

[2] G. Gutierrez and J. M. Yanez, Am. J. Phys. 65, 739 (1997).

[3] R. K. Pathria, Am. J. Phys. 66, 1080 (1998).

[4] W. S. Dai and M. Xie, Phys. Lett. A 311, 340 (2003).

[5] A. Sisman and I. Muller, Phys. Lett. A 320, 360 (2004).

[6] A. Sisman, J. Phys. A: Math. Gen. 37, 11353 (2004).

[7] W. S. Dai and M. Xie, Phys. Rev. E 70, 016103 (2004).

[8] W. S. Dai and M. Xie, Europhys. Lett. 72, 887 (2005).

[9] H. Pang, W. S. Dai, and M. Xie, J. Phys. A: Math. Gen. 39, 2563 (2006).

[10] A. Sisman, Z. F. Ozturk, and C. Firat, Phys. Lett. A 362, 16 (2007).

[11] W. S. Dai and M. Xie, J. Math. Phys. 48, 123302 (2007).

[12] W. S. Dai and M. Xie, Ann. Phys. 322, 1771 (2007).

[13] C. Firat and A. Sisman, Phys. Scr. 79, 065002 (2009).

[14] C. Firat, A. Sisman, and Z. F. Ozturk, Energy 35, 814 (2010).

[15] H. Pang, W. S. Dai, and M. Xie, J. Phys. A: Math. Theor. 44, 365001 (2011).

[16] A. Aydin and A. Sisman, Phys. Lett. A 378, 2001 (2014).

[17] S. Kjelstrup, S. K. Schnell, T. J. H. Vlugt, J. M. Simon, A. Bardow, D. Bedeaux, and T. Trinh, Adv. Nat. Sci.: Nanosci. Nanotechnol. 5, 023002 (2014).

[18] A. Aydin and A. Sisman, Phys. Scr. 90, 045208 (2015).

[19] A. Aydin and A. Sisman, Phys. Lett. A 380, 1236 (2016).

[20] A. Aydin and A. Sisman, Phys. Lett. A 382, 1807 (2018).

[21] A. Aydin and A. Sisman, Phys. Lett. A 383, 655 (2019).

[22] R. K. Pathria and P. D. Beale, Statistical Mechanics (Elsevier, Amsterdam, 2011).

[23] V. V. Mitin, D. I. Sementsov, and N. Z. Vagidov, Quantum Mechanics for Nanostructures (Cambridge University Press, New York, 2010).

[24] M. Lundstrom, Fundamentals of Carrier Transport (Cambridge University Press, New York, 2000).

[25] T. S. Fisher, Thermal Energy at the Nanoscale (World Scientific, Singapore, 2014).

[26] C. Weisbuch and B. Vinter, Quantum Semiconductor Structures (Academic, San Diego, 1991).
[27] M. D. Ventra, Electrical Transport in Nanoscale Systems (Cambridge University Press, Cambridge, UK, 2008).

[28] P. L. Butzer, P. J. S. G. Ferreira, G. Schmeisser, and R. L. Stens, Results Math. 59, 359 (2011).

[29] H. P. Baltes and E. R. Hilf, Spectra of Finite Systems (Bibliographisches Institut, Mannheim, 1976).

[30] H. Weyl, Nachr. Ges. Wiss. Gött. Math. Phys. K1. 2, 110 (1911).

[31] M. Kac, Am. Math. Mon. 73, 1 (1966).

[32] H. P. McKean Jr. and I. M. Singer, J. Diff. Geo. 1, 43 (1967).

[33] S. G. Samko, A. A. Kilbas, and O. Marichev, Fractional Integrals and Derivatives: Theory and Applications (Gordon and Breach, New York, 1993).

[34] R. Herrmann, Fractional Calculus: An Introduction for Physicists (World Scientific, New Jersey, 2014).

[35] K. B. Oldham and J. Spanier, The Fractional Calculus: Theory and Applications of Differentiation and Integration to Arbitrary Order (Dover, New York, 2002).

[36] L. Vazquez, J. J. Trujillo, and M. P. Velasco, Fract. Calc. Appl. Anal. 14, 334 (2011).

[37] D. Yin, R. Meng, X. Duan, and Y. Li, Physica A (Amsterdam, Neth.) 404, 1 (2014).

[38] Z. Korichi and M. T. Meftah, Theor. Math. Phys. 186, 374 (2016).

[39] R. A. Magomedov, R. R. Meilanov, R. P. Meilanov, E. N. Akhmedov, V. D. Beybalaev, and A. A. Aliverdiev, J. Therm. Anal. Calorim. 133, 1189 (2018).

[40] A. I. Saichev and W. Woyczynski, Distributions in the Physical and Engineering Sciences (Birkhäuser, New York, 2013), Vol. 2 .

[41] E. Camrud, Prog. Fract. Differ. Appl. 4, 463 (2018).

[42] C. G. Chakrabarti and I. Chakrabarty, Mod. Phys. Lett. B 20, 1471 (2006).

[43] T. Higuchi, Physica D (Amsterdam, Neth.) 31, 277 (1988).

Correction: The copyright license statement was presented incorrectly and has been fixed. 